\title{
TMEM147 promotes hepatocellular carcinoma progress by inducing EGFR/MAPK activity
}

Chengyi Sun ( $\nabla$ sunchengyi2014@163.com )

Guizhou Medical University https://orcid.org/0000-0002-8603-3609

Jing Xu

Guizhou Medical University

She Tian

Guizhou Medical University

Yanqing Liu

Guizhou Medical University

Changhao Zhu

Guizhou Medical University

Chao Yu

Guizhou Medical University

\section{Research}

Keywords: Hepatocellular carcinoma, TMEM147, EGFR, MAPK, glycolysis

Posted Date: March 4th, 2020

DOI: https://doi.org/10.21203/rs.3.rs-15788/v1

License: (c) This work is licensed under a Creative Commons Attribution 4.0 International License. Read Full License 


\section{Abstract}

Backgroud: Hepatocellular carcinoma (HCC) is characterized by rapid early proliferation and distant metastasis and is extremely difficult to treat. Aerobic glycolysis is a hallmark of abnormal glucose metabolism in cancer cells as has been shown to be associated with tumor proliferation and metastasis; however, the mechanisms underlying aerobic glycolysis remain unclear.

Methods: Immunohistochemistry (IHC) and qRT-PCR was performed to investigate the association between TMEM147 expression and the clinicopathological characteristics and prognosis of patients with HCC. Loss- and gain-of function assays were performed to investigate the role of TMEM147 in proliferation, metastasis and glycolysis in vitro and vivo. Bioinformatic analysis and rescue assay were used to demonstrated the TMEM147 interacted with EGFR and promoted its retromer-mediated recycling back to the plasma membrane.

Results: we identified TMEM147 as a protein that was highly expressed and associated with poor survival in patients with HCC. Both gain- and loss-of-function studies revealed that TMEM147 acted as a key oncoprotein by promoting HCC growth, metastasis, and glycolysis via the EGFR/ MAPK signaling pathway. Mechanistically, TMEM147 interacted with EGFR and promoted its retromer-mediated recycling back to the plasma membrane, thus increasing the stability of EGFR and prolonging activation of the downstream MAPK pathway.

Conclusion: Collectively, these results demonstrated the role and functional mechanism of TMEM147 in HCC, and indicated that TMEM147 may represent a prognostic biomarker and potential therapeutic target for HCC.

\section{Background}

Hepatocellular carcinoma (HCC) is the second-leading cause of cancer-related death worldwide[1]. Although improvements in many therapeutic strategies, including surgical resection, liver transplantation, and radiofrequency ablation, have improved the 5-year survival rate of HCC patients, its prognosis remains unsatisfactory[2]. Survival is associated with the use of targeted treatments, such as the tyrosine kinase inhibitor sorafenib, in patients with advanced stage cancer with vascular invasion and distant metastasis[3,4]. Clarifying the biological processes and molecular mechanisms underlying liver cancer progression might thus contribute to the effective treatment of HCC.

Although in the state of oxygen supply, tumor cells preferentially produce energy through aerobic glycolysis, rather than relying on mitochondria-mediated oxidative phosphorylation[5]. Aerobic glycolysis provides many of the metabolic precursors required for anabolism in rapidly proliferating cancer cells, such as the glycolysis intermediates 6-phosphate glucose and 6-phosphate fructose, which provide growth advantages for tumor cells[5, 6]. Previous studies have also shown that lactic acid is an important metabolite generated by the Warburg effect, which can promote cell migration, immune escape, and induce tumor metastasis[7,8]. Glycolysis therefore not only provides energy to tumor cells but also provides intermediate products required for cell biosynthesis, thus relieving metabolic pressure and providing a suitable environment and conditions for their rapid proliferation and distant metastasis $[9,10]$. However, although aerobic glycolysis in tumor cells has been shown to play important roles in various stages of cancer development, its association with tumor growth and metastasis remains elusive.

Accumulating evidence suggests that the mitogen-activated protein kinase (MAPK) pathway promotes glycolysis by transcriptionally regulating glucose metabolism-associated genes involved in aerobic glycolysis, ultimately enhancing cell proliferation and invasion abilities[11, 12]. Moreover, C-MYC, which is an essential transcription factor promoting glucose consumption and metabolism reprogramming, is a target gene of MAPK[13]. Epidermal growth factor receptor (EGFR) is the upstream target of MAPK and a well-known therapeutic target in various cancers, and has been shown to accelerate glucose consumption and lactate production in cancer cells, including HCC[14]. There is also growing evidence for the endosomal system as an essential site of MAPK signal transduction, with EGFR trafficking and recycling to the cell 
surface[15, 16]. Retromer has previously been reported to be responsible for the aberrant regulation of endosomal protein trafficking and recycling, leading to tumor progression[17]. Retromer is a highly conserved multimeric protein complex comprising a core cargo-recognition trimer composed of VPS26, VPS29, and VPS35[18, 19]. This therefore supports a pivotal role of EGFR trafficking and recycling in MAPK pathway-mediated aerobic glycolysis activation.

Transmembrane protein 147 (TMEM147), located in the intracellular compartment, acts as a regulator mediating receptor trafficking and has been identified as a possible oncoprotein in colon cancer[20,21]. However, its function and molecular mechanism in HCC remain unknown. In the current study, we showed that TMEM147 expression was markedly induced in HCC tissues and cell lines and was correlated with tumor size, metastasis, and poor survival of HCC patients. Both gainand loss-of-function studies determined that TMEM147 acted as a key oncogene by promoting HCC growth, metastasis, and glycolysis. Mechanistically, TMEM147 interacted with EGFR and facilitated its downstream MAPK signaling, thus mediating the progression and metabolic reprogramming of HCC.

\section{Materials And Methods}

\section{Clinical samples}

A total of 150 matched primary HCC and corresponding non-tumor tissues were collected from the Department of HepaticBiliary-Pancreatic Surgery, The Affiliated Hospital of Guizhou Medical University, between July 2010 and May 2019. The patients' diagnoses were verified by clinical and pathological features. All surgical specimens were frozen and stored at $80^{\circ} \mathrm{C}$ for further RNA and protein analyses, and were embedded in wax for IHC. This study was approved by the Ethics Committee of the Affiliated Hospital of Guizhou Medical University.

\section{Cell culture}

The human HCC cell lines and the human normal liver cell line used in this study were obtained from ATCC. All cells were cultured in Dulbecco's modified Eagle's medium (DMEM; Gibco, Carlsbad, CA, USA) supplemented with 10\% fetal bovine serum (FBS; $\mathrm{BI}$, Israel) and $1 \%$ penicillin (Beyotime, Shanghai, China) at $37^{\circ} \mathrm{C}$ in a humidified $5 \% \mathrm{CO}_{2}$ incubator.

\section{Western blot}

Western blots were performed as we protocol described and the protein extracted and detected materials were purchase from Beyotime. The antibodies used in this study were as follows: EGFR (Proteintech, 66455-1-lg), GLUT1 (Proteintech, 21829-1-AP), HK2 (Proteintech, 66974-1-lg), PKM (Proteintech, 10078-2-AP), MYC (Proteintech, 10828-1-AP), LDHA (Proteintech, 19987-1-AP), GAPDH (Proteintech, 60004-1-Ig), RAF (Abcam, ab200653), MEK (Abcam, ab96379), ERK (Abcam, ab17942), phosphorylated RAF (Abcam, ab4767), phosphorylated MEK (Abcam, ab96379), and phosphorylated ERK (Abcam, ab223500). Cycloheximide (CST, \#2112) was used to evaluate the protein stability of EGFR.

\section{RT-qPCR}

All RT-qPCR reagents were purchased from Takara (Japan). RNA was extracted from HCC cells using TRIzol and then reverse transcribed to produce cDNA for further quantitative detection. PCR amplifications for the quantification of TMEM147, GAPDH, GLUT1, HK2, PKM2, LDHA, and EGFR were performed using a SYBR kit. GAPDH was used as internal control (Table 1).

\section{Cell viability, proliferation, migration, and invasion assays}

CCK8, EDU, and colony-forming assays were used to measure cell viability and proliferation. For CCK8 assay, HCC cells were seeded in 96-well plates in DMEM containing 10\% FBS, and $200 \mu$ medium containing CCK-8 reagent was added to the wells. The optical density was then detected at 0, 24, 48, 72, and $96 \mathrm{~h}$. For the colony-formation assay, HCC cells were 
inoculated into culture dishes at 1000 cells/plate. After 2 weeks, the clones were fixed, stained, and counted under a microscope. For the EDU assay, HCC cells were seeded in a confocal dish at $2 \times 10^{5}$ cells/plate. The cells were then incubated with dye and stained with Hoechst 33342, followed by two washings with phosphate-buffered saline containing Tween-20. Images were captured using a microscope (Zeiss) at 400× magnification.

\section{In vivo assay}

For in vivo assay, TMEM147-overexpressing, TMEM147-knockdown, and NC SMMC-7721 cells were injected subcutaneously into BALB/c nude mice (4- 6 weeks old, females, $n=6$ per group). The mice were observed every week and euthanized 6 weeks after injection. The tumors were dissected and weighed. All conditions and procedures for the animal experiments were approved by the Animal Care Committee of Guizhou Medical University.

\section{Glucose consumption, lactate production, cellular ATP level, and ECAR}

Glucose consumption and lactate production were detected using assay kits (Beyotime). Glucose and lactate levels in the medium were measured using glucose and lactate assay kits, according to the respective instruction manuals. Glucose and lactate levels were normalized to the corresponding cell numbers. Cellular ATP levels were analyzed using a CellTiterGlo 2.0 Assay kit, according to the manufacturer's instructions. Relative intracellular ATP levels were normalized to the cell number of the corresponding cell lysate. The ECAR was determined using an XF96 Extracellular Flux analyzer (Seahorse Bioscience) according to the manufacturer's instructions, and the data were analyzed using Seahorse XF-96 Wave software. All values were normalized to cell number.

\section{Co-immunoprecipitation assay}

Co-immunoprecipitation assays were carried out using the following antibodies: TMEM147 (Abcam, ab97624), EGFR (Proteintech, 66455-1-lg), VPS26 (Proteintech, 12804-1-AP), VPS29 (Abcam, ab236796), and VPS35 (Proteintech, 10236-1AP). The indicated cell proteins were extracted using immunoprecipitation lysate buffer. After lysate quantification, $1 \mu \mathrm{g}$ of TMEM147 primary antibody or lgG was added to $500 \mu \mathrm{g}$ total protein lysate and shaken vertically overnight at $4^{\circ} \mathrm{C}$. Protein $A+G$ beads $25 \mu$ l were then added into the mixture and shaken vertically at $4^{\circ} \mathrm{C}$ for $4 \mathrm{~h}$. The beads were then washed three times using precooled immunoprecipitation buffer. Protein loading buffer $(2 \times)$ was mixed with the beads and boiled for 10 $\min$.

\section{IHC staining}

Tissue samples were fixed in $4 \%$ paraformaldehyde at $4^{\circ} \mathrm{C}$, sectioned into slices, and blocked in $5 \%$ bovine serum albumin in TBS for $2 \mathrm{~h}$ at room temperature. The primary antibodies were then added and incubated at $4^{\circ} \mathrm{C}$ overnight, followed by the secondary antibodies. The sections were subjected to DAB reaction. The sections were photographed using a digital microscope camera (Olympus, Tokyo, Japan).

\section{EdU incorporation assay}

The HCC cells were determined in vitro using the Cell-Light ${ }^{\mathrm{TM}}$ EdU staining kit (RiboBio, Guangzhou, China) according to manufacturer instructions. The proportion of positive cells in each well were counted.

\section{Immunofluorescence}

PC cells grown on the 6-well plates were fixed with $4 \%$ paraformaldehyde. Next, the cells were incubated with the primary antibody $(1: 100)$ at $4{ }^{\circ} \mathrm{C}$ overnight followed by

Alexa Fluor 594-conjugated goat anti-rabbit IgG (1:250) and FITC-conjugated goat anti-rabbit IgG (1:250). The slides were counterstained with DAPI to visualize cell nuclei. Images were recorded using a confocal lasers canning microscope. 


\section{Cell transfection and retrovirus transduction}

The lentiviruses and plasmids used in the study were constructed by and purchased from Genechem (Shanghai, China). Lentivirus transfection of HCC cells was carried out according to the polybrene protocol. Plasmids were transfected into HCC cells using Lipofectamine RNAiMAX (Thermo Fisher Scientific).

\section{Statistical analysis}

The data were analyzed and presented using GraphPad Prism 7.0 (GraphPad Software, La Jolla, CA, USA). Kaplan-Meier survival curves were constructed using SPSS software. Differences between groups were analyzed using two-tailed Student's $t$-tests and ANOVA. Results with $p<0.05$ were considered significant.

\section{Results}

\section{Association of TMEM147 expression with clinical outcomes in patients with HCC}

We evaluated TMEM147 expression in The Cancer Genome Atlas (TCGA) based on the RNA-seq data for liver hepatocellular carcinoma (LIHC). TMEM147 was highly expressed in HCC (Fig. 1A). TMEM147 expression levels were significantly elevated in HCC cell lines compared with normal liver cells, as demonstrated by reverse transcriptionquantitative polymerase chain reaction (RT-qPCR) and western blotting (Fig. 1B, C). We also determined the role of TMEM147 in an independent panel of primary HCC and adjacent normal tissues from patients with HCC by RT-qPCR and western blotting analysis. TMEM147 expression was upregulated in HCC tissues (Fig. 1D, E). To determine the clinical significance of TMEM147 expression in patients with HCC, we also analyzed its expression levels in a tissue microarray of 150 paired HCC samples with available clinical information. TMEM147 expression levels were compared between the HCC tumor samples and adjacent non-tumor tissues using immunohistochemistry (IHC). TMEM147 was overexpressed in tumor tissues compared with adjacent non-tumor tissues (Fig. 1F). IHC revealed high expression of TMEM147 in $71.3 \%$ $(107 / 150)$ of HCC patients and low expression in 28.7\% (43/150) of patients (Table 2). Moreover, TMEM147 expression levels were associated with tumor size $(p=0.024)$, serum alpha-fetoprotein (AFP) $(p=0.007)$ and vascular invasion ( $p=$ 0.028). However, there was no significant association between TMEM147 expression level and sex, age, TNM stage and tumor number. Based on TCGA data, survival analysis using the Kaplan-Meier method revealed that patients with low TMEM147 expression had significantly better overall survival (OS) $(p<0.001, \mathrm{HR}=2.1$, Fig. $1 \mathrm{G})$ and disease-free survival (DFS) $(p<0.05, H R=1.4$ Fig. 1H). Consistently, survival analysis in our cohort of 150 patients indicated that patients with low TMEM147 expression had better OS ( $<<0.05, \mathrm{HR}=1.6$, Fig. 1I). Overall, these results indicate that high TMEM147 expression was associated with a poorer prognosis in patients with HCC.

\section{Effects of TMEM147 on HCC growth and metastasis in vitro}

Given that TMEM147 was upregulated in HCC tissues and cell lines in our study, we investigated its role in HCC cells by gain- and loss-of-function studies. TMEM147-overexpressing (TMEM147U) and TMEM147-knockdown (TMEM147KD) lentivirus plasmids were transfected into SMMC-7721 and Hep3B cells and significantly upregulated and downregulated the expression levels of TMEM147, respectively, as shown by RT-qPCR and western blot (Fig. 2A, B). TMEM147overexpressing HCC cells demonstrated significantly faster growth according to CCK-8 assay (Fig. 2C). We also determined the role of TMEM147 in cell proliferation by EDU assay. The EDU-positivity rate was higher in TMEM147-overexpressing cells and lower in TMEM147-knockdown cells (Fig. 2D). Similarly, TMEM147 overexpression increased HCC colony formation (Fig. 2E), while TMEM147 knockdown significantly impaired growth and reduced colony formation compared with negative control (NC) cells. We also explored the role of TMEM147 in HCC cell migration and invasion by transwell assays. TMEM147-overexpressing cells showed higher migration and invasion activities while TMEM147-knockdown cells showed lower activities compared with control cells (Fig. 2F, G). 


\section{Effect of TMEM147 on glycolysis in HCC cells}

Gene Ontology (GO) enrichment analysis using TCGA database showed striking alterations in metabolic processes, including carbon metabolism and central carbon metabolism in cancer (Fig. 3A, Table 3), indicating a correlation between TMEM147 expression and enhanced carbon metabolism in HCC. Accordingly, aberrant glucose metabolism occurs in HCC and is known to be a vital metabolic process activating cancer cell proliferation and invasion. To verify the effect of TMEM147 on glycolysis, we examined the metabolic processes in TMEM147-overexpressing and -silenced HCC cells. Cellular glucose uptake and lactate production in the culture medium were significantly increased in TMEM147overexpressing cells (Fig. 3B, C), and TMEM147 overexpression was also associated with increased cellular ATP levels (Fig. 3D). In contrast, TMEM147 knockdown had the opposite effects. We further examined the effects of TMEM147 on glucose metabolism by measuring the extracellular acid ratio (ECAR). Silencing of TMEM147 attenuated whereas overexpression of TMEM147 enhanced the glycolytic capability of HCC cells, indicating that TMEM147 mainly affected the glycolytic component of glucose metabolism (Fig. 3E). Correlation analysis using TCGA database also showed that TMEM147 expression was positively related to several metabolic enzymes involved in glycolysis, including GLUT1, PKM2, HK1, HK2, LDHA, LDHA (Supplementary file 1: Fig. S1). Consistently, GLUT1, PKM2, HK2, LDHA were significantly downregulated by TMEM147 knockdown and upregulated by TMEM147 overexpression, as shown by RT-qPCR and western blot (Fig. 3F, G).

\section{Effect of TMEM147 on HCC growth in vivo}

We further evaluated the effects of TMEM147 on tumorigenesis in vivo in a mouse tumor xenograft model, by subcutaneously injecting NC, and TMEM147 NC (TMEM147NC), overexpressing (TMEM147U), and knockdown (TMEM147KD) SMMC-7721 cells into nude mice. Six representative xenograft tumors from each group were photographed at 6 weeks (Fig. 4A). TMEM147U increased tumor growth and volume compared with the NC group, whereas TMEMKD had the opposite effects (Fig. 4B, C). TMEM147 expression was positively correlated with glycolysis (GLUT1, HK2, PKM2, LDHA) and proliferation markers (Ki-67, proliferating cell nuclear antigen), as shown by IHC staining (Fig. 4D). Further, the hepatic metastasis model was used to evaluate the role of TMEM-147 in HCC cells metastasis ability. The TMEM-147 knockdown HepG2 cells were inoculated into nude spleen and then the HepG2 could reflow into the liver, the results demonstrated that TMEM147 knockdown group exerted less metastasis ability (Fig. 4E).

\section{Interaction between TMEM147 and EGFR and effect on MAPK signaling}

We elucidated the molecular mechanisms of TMEM147 in promoting HCC progression by Kyoto Encyclopedia of Genes and Genomes (KEGG) pathway analysis. TMEM147 was correlated with MAPK activation by receptor tyrosine kinases (RTKs) (Supplementary file 2: Fig. S2). Considering that EGFR is one of the RTKs that activate the MAPK signal pathway, we hypothesized that TMEM147 might exert its biological function via the downstream EGFR/MAPK signaling pathway. We verified the interaction between TMEM147 and EGFR by immunoprecipitation assay and showed that TMEM147 could combine with EGFR (Fig. 5A), and further demonstrated their colocalization by immunofluorescence (Fig. 5B). Furthermore, EGFR expression and MAPK activity were both upregulated in TMEM147-overexpressing cells and inhibited in TMEM147downregulated cells, as shown by western blot (Fig. 5C). To confirm the correlation between TMEM147 and EGFR, we added EGFR-silencing RNA (sh-EGFR) and an EGFR inhibitor (sorafenib) to TMEM147-overexpressing HCC cells. TMEM147 significantly promoted EGFR/MAPK activation, while this effect was reversed by either sh-EGFR or sorafenib (Fig. 5D). We verified the correlation between TMEM147 expression and EGFR/MAPK activity by analyzing the expression of TMEM147, EGFR, phosphorylated ERK, phosphorylated MEK, and phosphorylated RAF, and showed that TMEM147 expression was positively correlated with EGFR/MAPK pathway hub genes (Fig. 5E).

\section{TMEM147 drives HCC tumorigenesis through EGFR}


We verified EGFR as the functional interactor driving HCC tumorigenesis by examining the effects of EGFR shRNA and the EGFR inhibitor sorafenib. Downregulation and inhibition of EGFR attenuated the increased proliferation caused by TMEM147 overexpression, as shown by CCK8, EDU, and colony-formation assays (Fig. 6A-C). EGFR knockdown and inhibition also partly restrained the effect of TMEM147 overexpression on PC cell migration and invasion in transwell assays (Fig. 6D, E). To verify the role of EGFR in the TMEM147-mediated glycolysis effect, we demonstrated that TMEM147 overexpression induced glucose uptake, lactate production, and cellular ATP levels, and these effects could be rescued by EGFR downregulation or inhibition (Fig. 6F-H). Furthermore, EGFR downregulation or inhibition reduced the TMEM147-induced expression of key glycolysis enzymes at both the mRNA and protein levels, as shown by RT-qPCR and western blot (Fig. 6I, J).

\section{Role of retromer in TMEM147-induced EGFR/ MAPK pathway}

TMEM147 was previously shown to localize to endoplasmic reticulum membranes and the Golgi apparatus, as a regulator of M3R trafficking to the cell surface[21]. However, retromer is a highly conserved protein complex important for EGFR trafficking and subcellular organelle location from the endosomes to trans-Golgi network or plasma membrane[22, 23]. We therefore proposed that a hypothetical retromer complex might act as a platform for TMEM147-mediated EGFR subcellular organelle localization, trafficking, and activity regulation. Correlation analysis using TCGA database showed that TMEM147 expression was positively related to the retromer components, including VPS26, VPS29, VPS35(Fig. 7A). We therefore explored the possible involvement of retromer complex structures in TMEM47-induced EGFR/MAPK signaling. We determined if TMEM147 and retromer influenced EGFR trafficking to the membrane by EGFR recycling assay. EGFR recycling was increased compared with the NC, and this effect was reversed by downregulation of VPS26, VPS29, or VPS35 (Fig.7B). And the result was verified by immunofluorescence assay (Fig. 7C). Furthermore, EGFR degradation was slower in TMEM147-overexpressing cells and faster in VPS26-, VPS29-, and VPS35-downregulated HCC cells, according to protein stability assay (Fig. 7D). We subsequently evaluated the role of the TMEM147/retromer axis in the EGFR downstream MAPK pathway by western-blot assay. TMEM47 overexpression significantly activated the MAPK pathway and upregulated phosphorylated ERK, MEK, and MEK expression, while this effect was reversed by downregulation of VPS26, VPS29, or VPS35 (Fig. 7E).

\section{Effects of TMEM147 on HCC proliferation, invasion, and glycolysis via retromer components}

We determined if TMEM147 induced HCC proliferation, invasion, and glycolysis via the retromer components VPS26, VPS29, and VPS35 by examining the effects of VPS26, VPS29, and VPS35 shRNAs. VPS26, VPS29, and VPS35 downregulation attenuated the TMEM147-induced proliferation, detected by CCK8 and colony-formation assays (Fig. 8A, B). Furthermore, VPS26, VPS29, and VPS35 knockdown partly reversed the effects of TMEM147 overexpression on HCC cell migration and invasion in transwell assays (Fig. 8C, D). We investigated the role of the retromer components in the TMEM147-mediated glycolysis effect by bioinformatics analysis using TCGA database. VPS26, VPS29, and VPS35 expression were positively related to several metabolic enzymes involved in glycolysis, including GLUT1, PKM2, HK1, HK2, LDHA, and LDHA (Supplementary file 3: Fig. S3). TMEM147 overexpression induced glucose uptake and lactate production, while these effects were rescued by VPS26, VPS29, or VPS35 downregulation (Fig. 8E-F).

\section{Discussion}

In this study, we examined the regulatory role of TMEM147 in the proliferation, invasion, and aerobic glycolysis of HCC cells. TMEM147 was upregulated in HCC, and its expression was positively correlated with a poorer prognosis in patients with HCC. Functional studies revealed that TMEM147 overexpression significantly promoted HCC cell proliferation, migration, and invasion, both in vivo and in vitro. Furthermore, bioinformatics analysis using TCGA revealed a correlation between TMEM147 expression and enhanced carbon metabolism in HCC. We therefore considered that TMEM147 might be a critical regulator of aberrant glucose metabolism in HCC. 
Accumulating studies have enhanced our understanding of the molecular mechanisms underlying the metabolic reprogramming associated with HCC progression; however, the role of aerobic glycolysis in cancer cell growth and metastasis remains unclear[24, 25]. We therefore explored the effect of TMEM147 on HCC progression and revealed the mechanism underlying the EGFR/MAPK signaling pathway. We showed that TMEM147 promoted cancer cell tumorigenesis and aerobic glycolysis in HCC. TMEM147 was also reported to contribute to colon cancer cell progression, based on bioinformatics and experimental verification[20]. However, information on the mechanism of TMEM147 in cancer progression is lacking. We therefore examined the downstream pathway involved in TMEM147-induced HCC proliferation, invasion, and glycolysis, and showed that RTK-mediated MAPK signal pathway activation was enriched by TMEM147 in HCC. EGFR is known to be an important oncoprotein in HCC, and provides a therapeutic target for drugs such as sorafenib[26]. Activation of EGFR by ligands such as EGF can mediate abnormal activation of multiple signaling pathways in cancer cells, most notably the MAPK pathway[27]. We focused on the interaction between TMEM147 and EGFR in HCC cells, and demonstrated that TMEM147 combined with EGFR and colocalized in the cytoplasm, using immunoprecipitation and immunofluorescence experiments. Furthermore, TMEM147 activated the MAPK pathway, as shown by western blotting and verified by IHC in HCC tissues. The MAPK signaling pathway is closely related to tumor progression, and its transcriptional regulation of the cell cycle mediates proliferation, and activation of matrix metalloproteinases to promote invasion and metastasis[28,29]. We confirmed that TMEM147 could bind to EGFR and regulate the downstream MAPK pathway, and further investigated if TMEM147 could affect the growth, metastasis, and glycolysis of HCC cells through EGFR. EGFR knockdown or inhibition abrogated the functional influences of TMEM147 on HCC cells, suggesting that TMEM147 not only binds to EGFR and activates the downstream MAPK pathway, but also exerts its tumorigenic functions in HCC through EGFR.

We further explored the mechanisms by which TMEM147 activated EGFR and MAPK in HCC cells by determining if the TMEM147-induced increase in EGFR levels was attributable to increased protein stability. Neither TMEM147 upregulation nor downregulation affected EGFR mRNA levels; however, western blotting showed that TMEM147 overexpression promoted EGFR protein stability and expression, suggesting that TMEM147 increased EGFR expression levels via posttranscriptional regulation. TMEM147 is located in the Golgi and intracellular compartment and acts as a regulator mediating M3R trafficking and cellular location[21]. We therefore determined if TMEM147 could affect trafficking in the subcellular structure. Retromer was previously identified as an endosome-independent complex essential for protein trafficking from the cytoplasm to the plasma membrane[30]. We therefore hypothesized that TMEM147 might mediate EGFR transport via the retromer complex, affecting its cellular localization and protein stability, and ultimately activating the downstream MAPK signaling pathway. The retromer components VPS26, VPS29, and VPS35 were positively correlated with glycolysis enzymes, consistent with the effect of TMEM147. To confirm if TMEM147 promoted EGFR trafficking and expression levels via the retromer complex, we performed rescue experiments with VPS26, VPS29, and VPS35 shRNAs, and showed that downregulation of these retromer components inhibited the proliferation, invasion, and glycolysis enhanced

by TMEM147. Furthermore, the TMEM147-induced increases in EGFR expression and MAPK activity were also inhibited by VPS26, VPS29, and VPS35 knockdown. To confirm if the retromer was an essential platform for TMEM147-induced EGFR trafficking and recycling, we performed an EGFR recycling assay during retromer inhibition, and demonstrated that TMEM147 promoted EGFR expression and the MAPK signal pathway through the retromer complex.

\section{Conclusions}

Overall, the results of this study identified TMEM147 as a novel oncoprotein responsible for potentiating proliferation, metastasis, and glycolysis and thus enhancing the progression of HCC. Regarding the mechanism, we also demonstrated that TMEM147 combines with EGFR to activate the downstream MAPK pathway in HCC. This interaction involves the retromer complex as a platform, mediating EGFR recycling to the cell membrane and enhancing EGFR protein stability.

\section{Abbreviations}

Page 8/22 
TMEM147: transmembrane protein 147; EGFR: epidermal growth factor receptor; MAPK: mitogen-activated protein kinase ; HCC: Hepatocellular carcinoma ; qRT-PCR: Real-time quantitative reverse transcription PCR; CCK-8: Cell counting kit; TMEM147U: TMEM147-overexpressing and TMEM147-upregulated ; NC: negative control; TMEM147U+sh-VPS26: TMEM147-upregulated combined with knockdown of VPS26; TMEM147U+sh-VPS29: TMEM147-upregulated combined with knockdown of VPS29; TMEM147U+sh-VPS35: TMEM147-upregulated combined with knockdown of VPS35

\section{Declarations}

\section{Ethics approval and consent to participate}

This study was reviewed and approved by the Medical Ethics Committee of The Affiliated Hospital of Guizhou Medical University, Guiyang, China. All animal studies were approved by the Institutional Animal Care and Use Committee of Committee of he Affiliated Hospital of Guizhou Medical University.

\section{Consent for publication}

All authors have agreed to publish this manuscript.

\section{Availability of data and material}

The datasets used and/or analyzed during the current study are available from the corresponding author on reasonable request.

\section{Competing interests}

The authors declare that they have no competing interests.

\section{Funding}

This work was supported by grants from the National Natural Science Foundation of China (81560477 and 81860505 to Chengyi Sun, 81660483 to Chao Yu).

\section{Authors' contributions}

Chengyi Sun and Chao Yu contributed to the experiment design, and data analysis. Jing Xu, She Tian and Yanqing Liu contributed to the experiment implementation, Changhao Zhu and Shiyu Chen ontributed to manuscript draft and data analysis. All authors read and approved the final manuscript.

\section{Acknowledgments:}

We thank Susan Furness, PhD, from Liwen Bianji, Edanz Editing China (www.liwenbianji.cn/ac), for editing the English text of a draft of this manuscript.

\section{References}

1. Siegel RL, Miller KD, Jemal A: Cancer statistics, 2019. CA Cancer J Clin 2019, 69:7-34.

2. Fukami Y, Kaneoka Y, Maeda A, Kumada T, Tanaka J, Akita T, Kubo S, Izumi N, Kadoya M, Sakamoto M, et al: Liver Resection for Multiple Hepatocellular Carcinomas: A Japanese Nationwide Survey. Ann Surg 2019.

3. Ohki T, Kondo M, Karasawa Y, Kawamura S, Maeshima S, Kojima K, Seki M, Toda N, Shioda Y, Tagawa K: Erratum to: Evaluation of the Efficacy of Sorafenib on Overall Survival in Patients with Hepatocellular Carcinoma using FT Rate: A Devised Index. Adv Ther 2017, 34:1773-1774. 
4. Kok VC, Chen YC, Chen YY, Su YC, Ku MC, Kuo JT, Yoshida GJ: Sorafenib with Transarterial Chemoembolization Achieves Improved Survival vs. Sorafenib Alone in Advanced Hepatocellular Carcinoma: A Nationwide PopulationBased Cohort Study. Cancers (Basel) 2019, 11.

5. Sosa V, Moline T, Somoza R, Paciucci R, Kondoh H, LLeonart ME: Oxidative stress and cancer: an overview. Ageing Res Rev 2013, 12:376-390.

6. Lunt SY, Vander HM: Aerobic glycolysis: meeting the metabolic requirements of cell proliferation. Annu Rev Cell Dev Biol 2011, 27:441-464.

7. Kathagen-Buhmann A, Maire CL, Weller J, Schulte A, Matschke J, Holz M, Ligon KL, Glatzel M, Westphal M, Lamszus $\mathrm{K}$ : The secreted glycolytic enzyme GPI/AMF stimulates glioblastoma cell migration and invasion in an autocrine fashion but can have anti-proliferative effects. Neuro Oncol 2018, 20:1594-1605.

8. Ganapathy-Kanniappan S: Linking tumor glycolysis and immune evasion in cancer: Emerging concepts and therapeutic opportunities. Biochim Biophys Acta Rev Cancer 2017, 1868:212-220.

9. Tan VP, Miyamoto S: HK2/hexokinase-II integrates glycolysis and autophagy to confer cellular protection. Autophagy 2015, 11:963-964.

10. Shi Y, Liu S, Ahmad S, Gao Q: Targeting Key Transporters in Tumor Glycolysis as a Novel Anticancer Strategy. Curr Top Med Chem 2018, 18:454-466.

11. Papa S, Choy PM, Bubici C: The ERK and JNK pathways in the regulation of metabolic reprogramming. Oncogene 2019, 38:2223-2240.

12. Dimri M, Humphries A, Laknaur A, Elattar S, Lee TJ, Sharma A, Kolhe R, Satyanarayana A: NAD(P)H Quinone Dehydrogenase 1 Ablation Inhibits Activation of the Phosphoinositide 3-Kinase/Akt Serine/Threonine Kinase and Mitogen-Activated Protein Kinase/Extracellular Signal-Regulated Kinase Pathways and Blocks Metabolic Adaptation in Hepatocellular Carcinoma. Hepatology 2019.

13. Qin Y, Hu Q, Ji S, Xu J, Dai W, Liu W, Xu W, Sun Q, Zhang Z, Ni Q, et al: Homeodomain-interacting protein kinase 2 suppresses proliferation and aerobic glycolysis via ERK/cMyc axis in pancreatic cancer. Cell Prolif 2019, 52:e12603.

14. Yin Y, Sun M, Zhan X, Wu C, Geng P, Sun X, Wu Y, Zhang S, Qin J, Zhuang Z, Liu Y: EGFR signaling confers resistance to BET inhibition in hepatocellular carcinoma through stabilizing oncogenic MYC. J Exp Clin Cancer Res 2019, 38:83.

15. Ren Y, Cheng L, Rong Z, Li Z, Li Y, Zhang X, Xiong S, Hu J, Fu XY, Chang Z: hSef potentiates EGF-mediated MAPK signaling through affecting EGFR trafficking and degradation. Cell Signal 2008, 20:518-533.

16. Dutta S, Roy S, Polavaram NS, Stanton MJ, Zhang H, Bhola T, Honscheid P, Donohue TJ, Band H, Batra SK, et al: Neuropilin-2 Regulates Endosome Maturation and EGFR Trafficking to Support Cancer Cell Pathobiology. Cancer Res 2016, 76:418-428.

17. McNally KE, Faulkner R, Steinberg F, Gallon M, Ghai R, Pim D, Langton P, Pearson N, Danson CM, Nagele H, et al: Retriever is a multiprotein complex for retromer-independent endosomal cargo recycling. Nat Cell Biol 2017, 19:12141225.

18. Zhang HX, Xu ZS, Lin H, Li M, Xia T, Cui K, Wang SY, Li Y, Shu HB, Wang YY: TRIM27 mediates STAT3 activation at retromer-positive structures to promote colitis and colitis-associated carcinogenesis. Nat Commun 2018, 9:3441.

19. Wang J, Fedoseienko A, Chen B, Burstein E, Jia D, Billadeau DD: Endosomal receptor trafficking: Retromer and beyond. Traffic 2018, 19:578-590.

20. Feng Y, Li Y, Li L, Wang X, Chen Z: Identification of specific modules and significant genes associated with colon cancer by weighted gene coexpression network analysis. Mol Med Rep 2019, 20:693-700.

21. Rosemond E, Rossi M, McMillin SM, Scarselli M, Donaldson JG, Wess J: Regulation of M(3) muscarinic receptor expression and function by transmembrane protein 147. Mol Pharmacol 2011, 79:251-261. 
22. Olivares-Castineira I, Llimargas M: EGFR controls Drosophila tracheal tube elongation by intracellular trafficking regulation. Plos Genet 2017, 13:e1006882.

23. Priya A, Sugatha J, Parveen S, Lacas-Gervais S, Raj P, Gilleron J, Datta S: Essential and selective role of SNX12 in transport of endocytic and retrograde cargo. J Cell Sci 2017, 130:2707-2721.

24. Liu R, Li Y, Tian L, Shi H, Wang J, Liang Y, Sun B, Wang S, Zhou M, Wu L, et al: Gankyrin drives metabolic reprogramming to promote tumorigenesis, metastasis and drug resistance through activating beta-catenin/c-Myc signaling in human hepatocellular carcinoma. Cancer Lett 2019, 443:34-46.

25. Kim J, Yu L, Chen W, Xu Y, Wu M, Todorova D, Tang Q, Feng B, Jiang L, He J, et al: Wild-Type p53 Promotes Cancer Metabolic Switch by Inducing PUMA-Dependent Suppression of Oxidative Phosphorylation. Cancer Cell 2019, 35:191203.

26. Niu L, Liu L, Yang S, Ren J, Lai P, Chen GG: New insights into sorafenib resistance in hepatocellular carcinoma: Responsible mechanisms and promising strategies. Biochim Biophys Acta Rev Cancer 2017, 1868:564-570.

27. Lin CC, Suen KM, Stainthorp A, Wieteska L, Biggs GS, Leitao A, Montanari CA, Ladbury JE: Targeting the Shc-EGFR interaction with indomethacin inhibits MAP kinase pathway signalling. Cancer Lett 2019, 457:86-97.

28. Rezatabar S, Karimian A, Rameshknia V, Parsian H, Majidinia M, Kopi TA, Bishayee A, Sadeghinia A, Yousefi M, Monirialamdari M, Yousefi B: RAS/MAPK signaling functions in oxidative stress, DNA damage response and cancer progression. J Cell Physiol 2019.

29. Del BBI, Nebreda AR: Roles of p38 MAPKs in invasion and metastasis. Biochem Soc Trans 2012, 40:79-84.

30. McNally KE, Cullen PJ: Endosomal Retrieval of Cargo: Retromer Is Not Alone. Trends Cell Biol 2018, 28:807-822.

\section{Tables}

Table 1. Sequences of primers used for real-time PCR Association between TMEM147 expression and clinical outcomes of HCC patients

\begin{tabular}{lll}
\hline Gene & \multicolumn{1}{c}{ Forward Primer } & Reverse Primer \\
TMEM147 & 5'-ACACGCTATGATCTGTACCACA-3' & 5'-CAGAGGTGGACGAAGGTCTC-3' \\
GLUT1 & 5'-GGCCAAGAGTGGCTAAAGAA-3' & 5'-ACAGCGTTGATGCCAGACAG-3 \\
HK2 & 5'-GAGCCACCACTCACCCTACT-3' & 5'-CCAGGCATTCGCAATGTG-3' \\
PKM2 & 5'-ATGTCGAAGCCCCATAGTGAA-3' & 5'-TGGGTGGTGATCAATGTCCA-3' \\
LDHA & 5'-ATGGCAACTCTAAAGGATCAGC-3'' & 5'-CCAACCCCAACAACTGTAATCT-3' \\
PDK1 & 5'-CTGTGATACGGATCAGAACCCG-3' & 5'-TCCACCAAACAATAAAGAGTGCT-3' \\
LDHB & 5'-TGGTATGGCGTGTGCTATCAG -3' & 5'-TTGGCGGTCACAGAATAATCTTT -3' \\
GAPDH & 5'-GGAGCGAGATCCCTCCAAAAT -3' & 5'-GGCTGTTGTCATACTTCTCATGG -3' \\
\hline
\end{tabular}

Table 2. Association between TMEM147 expression and clinical outcomes of HCC patients 


\begin{tabular}{|c|c|c|c|c|}
\hline \multicolumn{5}{|c|}{ Association of TMEM147 expression with Clinicopathologic Features } \\
\hline \multirow[t]{2}{*}{ Features } & \multirow[t]{2}{*}{ Total } & \multicolumn{2}{|c|}{ TMEM147 Expression } & \multirow[t]{2}{*}{$P$ value } \\
\hline & & Low(43) & $\operatorname{High}(107)$ & \\
\hline Sex & & & & 0.826 \\
\hline Male & 99 & 29 & 70 & \\
\hline Female & 51 & 14 & 37 & \\
\hline Age (years) & & & & 0.607 \\
\hline$\leq 50$ & 68 & 20 & 48 & \\
\hline$>50$ & 82 & 23 & 59 & \\
\hline Serum AFP (ng/ml)* & & & & 0.007 \\
\hline$<400$ & 48 & 29 & 19 & \\
\hline$\geq 400$ & 102 & 14 & 88 & \\
\hline HbsAg & & & & 0.654 \\
\hline Negative & 13 & 5 & 8 & \\
\hline Positive & 137 & 38 & 99 & \\
\hline Tumor size $(\mathrm{cm}) *$ & & & & 0.024 \\
\hline$\leq 5$ & 49 & 23 & 26 & \\
\hline$>5$ & 101 & 20 & 81 & \\
\hline Tumor number & & & & 0.687 \\
\hline Single & 47 & 15 & 32 & \\
\hline Multiple & 103 & 28 & 75 & \\
\hline TNM & & & & 0.129 \\
\hline I/II & 109 & 32 & 77 & \\
\hline III/IV & 41 & 11 & 30 & \\
\hline \multicolumn{5}{|l|}{ Vascular invasion * } \\
\hline Yes & 116 & 34 & 82 & 0.028 \\
\hline No & 34 & 9 & 25 & \\
\hline
\end{tabular}

Table 3. Gene Ontology (GO) enrichment analysis using TCGA database analysis of the pathway in the liver cancer 


\begin{tabular}{|c|c|c|c|c|c|c|c|c|c|}
\hline Category & $\%$ & PValue & $\begin{array}{l}\text { List } \\
\text { Total }\end{array}$ & $\begin{array}{l}\text { Pop } \\
\text { Hits }\end{array}$ & $\begin{array}{l}\text { Pop } \\
\text { Total }\end{array}$ & $\begin{array}{l}\text { Fold } \\
\text { Enrichment }\end{array}$ & Bonferroni & Benjamini & FDR \\
\hline Ribosome & 0.033099 & $2.87 \mathrm{E}-09$ & 136 & 144 & 6750 & 6.204044 & 5.17E-07 & $5.17 \mathrm{E}-07$ & $3.54 \mathrm{E}-06$ \\
\hline Spliceosome & 0.022066 & $4.85 \mathrm{E}-05$ & 136 & 129 & 6750 & 4.616963 & 0.008701 & 0.00436 & 0.059785 \\
\hline $\begin{array}{l}\text { Valine, leucine } \\
\text { and isoleucine } \\
\text { degradation }\end{array}$ & 0.007355 & 0.070564 & 136 & 48 & 6750 & 4.136029 & 0.999998 & 0.807276 & 59.39841 \\
\hline $\begin{array}{l}\text { Central carbon } \\
\text { metabolism in } \\
\text { cancer }\end{array}$ & 0.009194 & 0.034977 & 136 & 62 & 6750 & 4.002609 & 0.998353 & 0.656341 & 35.5026 \\
\hline $\begin{array}{l}\text { Ribosome } \\
\text { biogenesis in } \\
\text { eukaryotes }\end{array}$ & 0.011033 & 0.020567 & 136 & 79 & 6750 & 3.769546 & 0.976264 & 0.526758 & 22.58432 \\
\hline $\begin{array}{l}\text { Non-alcoholic } \\
\text { fatty liver } \\
\text { disease } \\
\text { (NAFLD) } \\
\end{array}$ & 0.018388 & 0.002746 & 136 & 148 & 6750 & 3.353537 & 0.390381 & 0.152084 & 3.330055 \\
\hline $\begin{array}{l}\text { FoxO } \\
\text { signaling } \\
\text { pathway }\end{array}$ & 0.01471 & 0.015689 & 136 & 131 & 6750 & 3.030983 & 0.941943 & 0.509132 & 17.69803 \\
\hline $\begin{array}{l}\text { Thyroid } \\
\text { hormone } \\
\text { signaling } \\
\text { pathway }\end{array}$ & 0.011033 & 0.075475 & 136 & 113 & 6750 & 2.635346 & 0.999999 & 0.791854 & 61.96346 \\
\hline $\begin{array}{l}\text { Carbon } \\
\text { metabolism }\end{array}$ & 0.011033 & 0.075475 & 136 & 113 & 6750 & 2.635346 & 0.999999 & 0.791854 & 61.96346 \\
\hline $\begin{array}{l}\text { Regulation of } \\
\text { actin } \\
\text { cytoskeleton }\end{array}$ & 0.016549 & 0.057691 & 136 & 209 & 6750 & 2.137278 & 0.999977 & 0.783032 & 51.9023 \\
\hline $\begin{array}{l}\text { Huntington's } \\
\text { disease }\end{array}$ & 0.01471 & 0.087716 & 136 & 191 & 6750 & 2.078842 & 年 & 0.808425 & 67.72244 \\
\hline $\begin{array}{l}\text { Viral } \\
\text { carcinogenesis }\end{array}$ & 0.01471 & 0.089591 & 136 & 192 & 6750 & 2.068015 & 1 & 0.78474 & 68.52995 \\
\hline
\end{tabular}

Figures 
A.

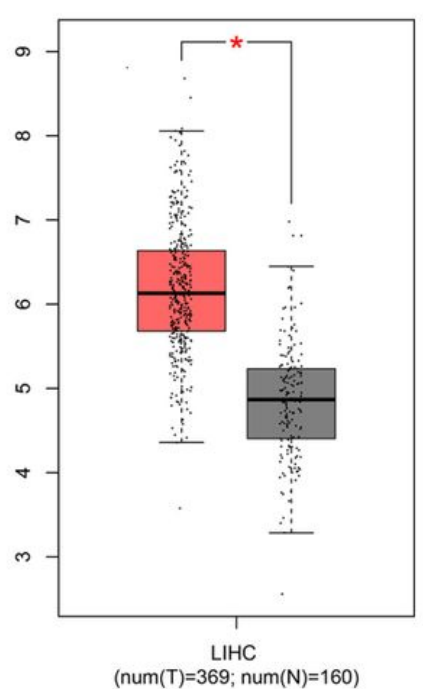

E

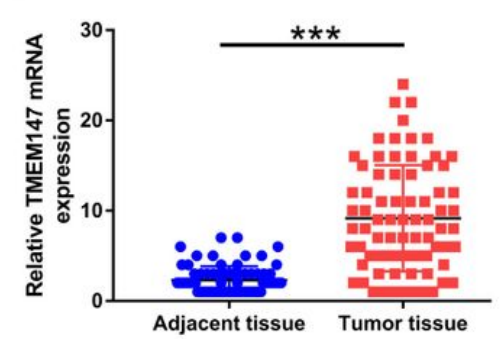

B.

D.
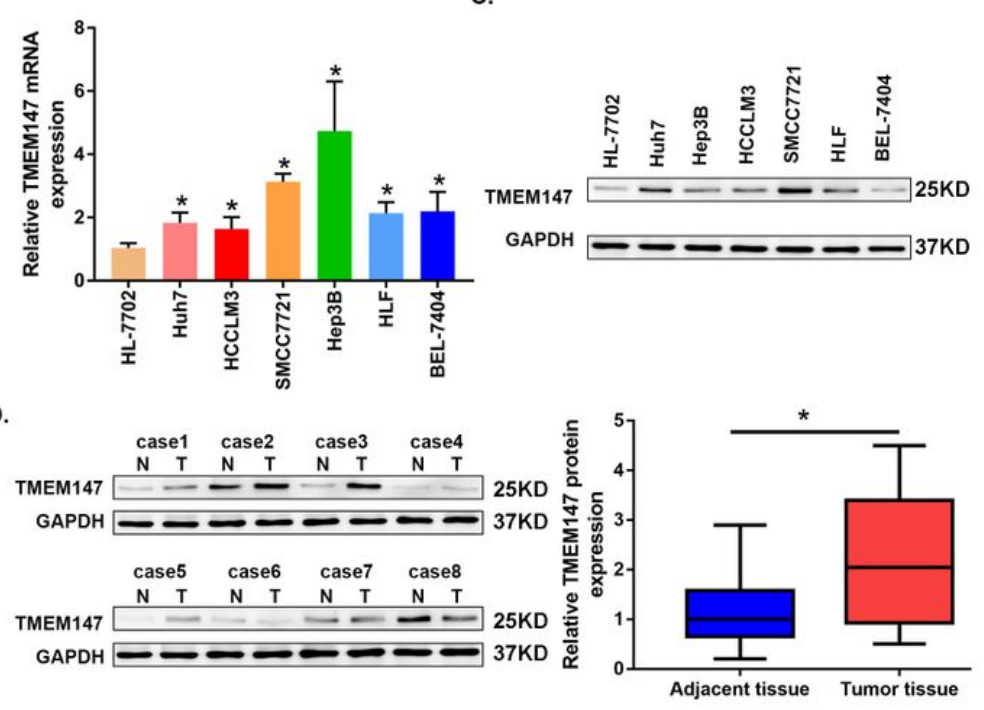

G.

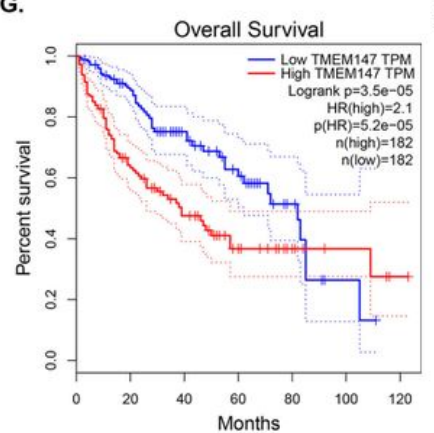

F.

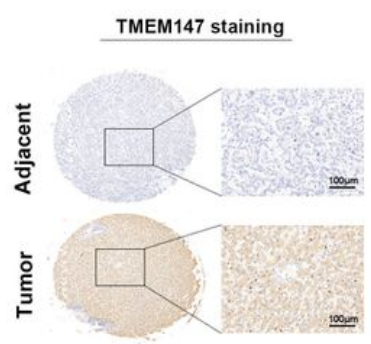

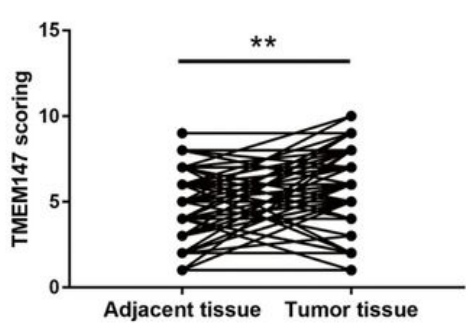

Adjacent tissue Tumor tissue

H.

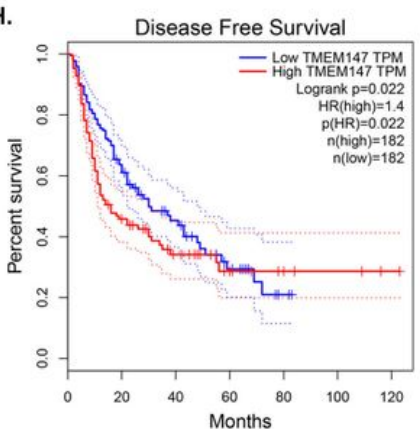

I.

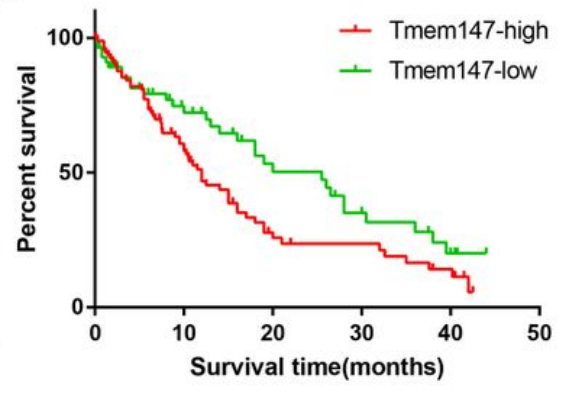

Figure 1

Association between TMEM147 expression and clinical outcome in HCC patients a. TMEM147 expression level in LIHC in TCGA database. b, c. RT-qPCR and western blot analyses of TMEM147 expression in one normal liver and six HCC cell lines. d, e. RT-qPCR and western blot analyses of TMEM147 expression in primary HCC and adjacent normal tissues. F. IHC analysis of tissue microarray containing 75 paired HCC and normal samples. g, h. Kaplan-Meier survival analysis of TMEM147 expression in relation to OS and DFS in HCC based on TCGA database. i. Kaplan-Meier survival analysis of TMEM147 expression in relation to survival in our cohort of $75 \mathrm{HCC}$ patients. 


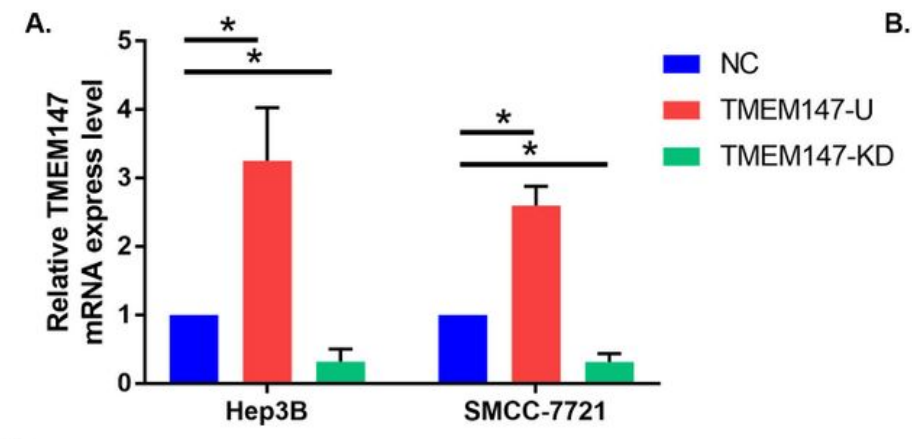

B.

c.
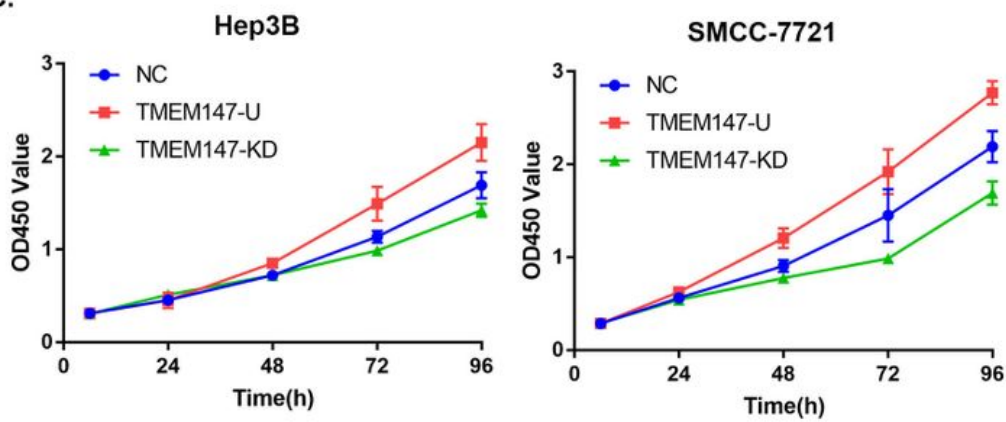

D.
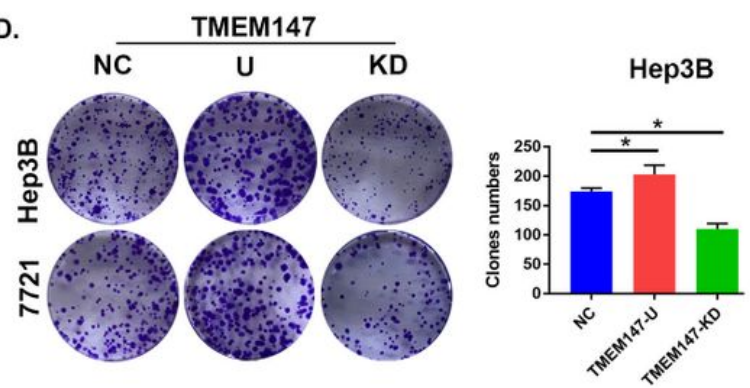

F.

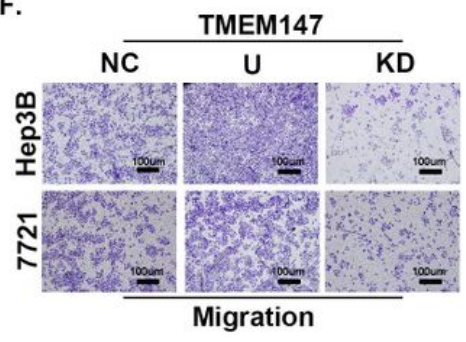

G.

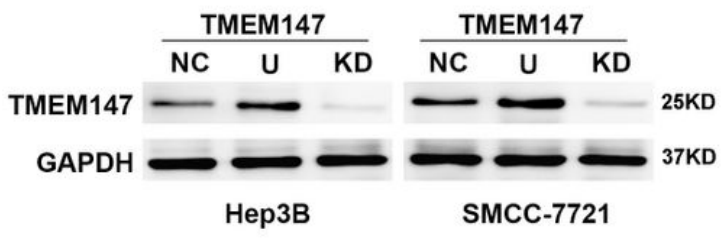

E.

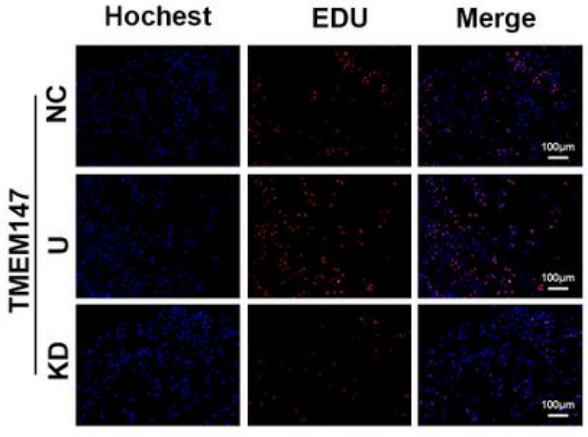

Нер3в
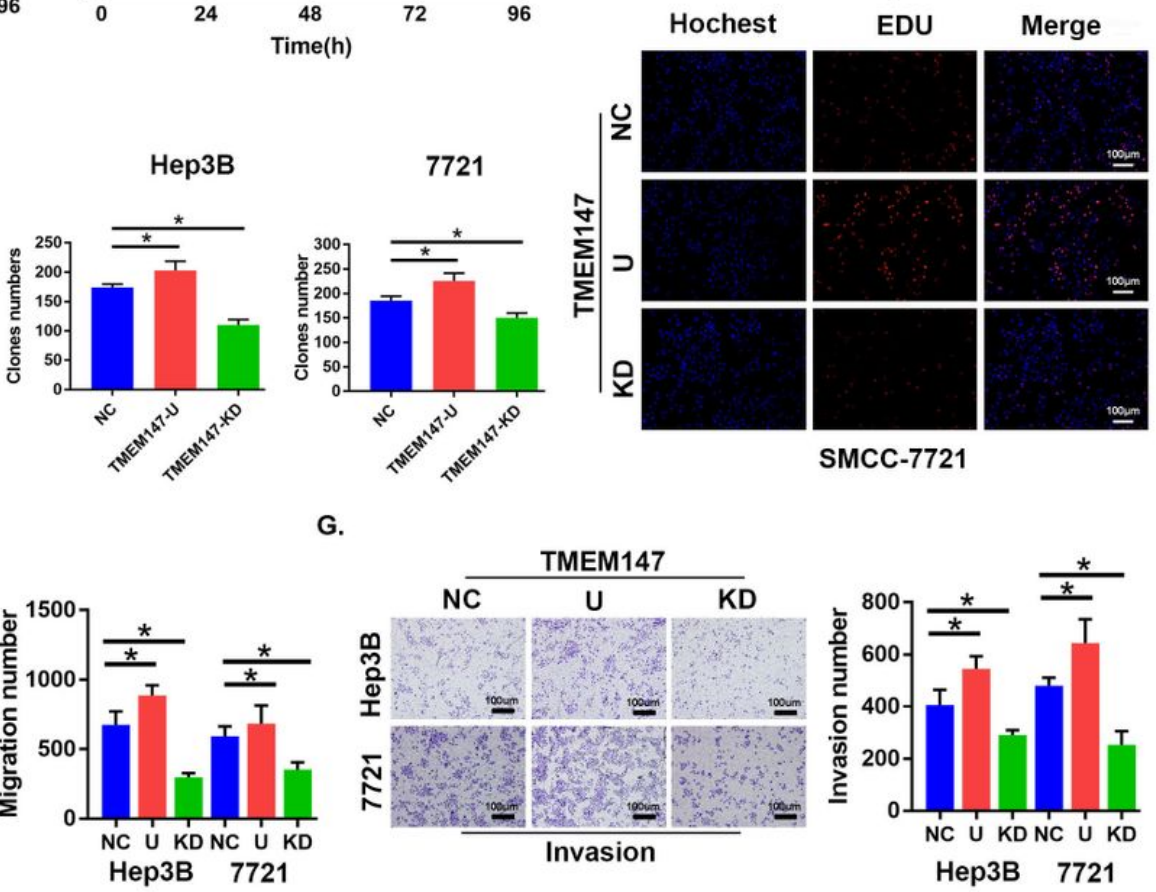

Figure 2

Effects of TMEM147 on HCC growth and metastasis in vitro a, b. Transfection efficiency of TMEM147-overexpressing (TMEM147U) and TMEM147-knockdown (TMEM147KD) lentivirus plasmids in HCC cell lines according to RT-qPCR and western blot. c. The viabilities of SMMC-7721 and Hep3B cells transduced with TMEM147-overexpressing and -knockdown lentiviruses, respectively, determined by CCK-8 assay. d. Colony-formation of SMMC-7721 and Hep3B cells transduced with TMEM147-overexpressing and -knockdown lentiviruses, respectively. e. Growth of SMMC-7721 and Hep3B cells transduced with TMEM147-overexpressing and -knockdown lentiviruses, respectively, determined by EDU assay. $\mathrm{f}, \mathrm{g}$. In vitro migration and invasion abilities of SMMC-7721 and Hep3B cells transduced with TMEM147-overexpressing and knockdown lentiviruses, respectively, determined by transwell migration and invasion assays. ${ }^{*} p<0.05$ was regarded as statistically significant. 
A.

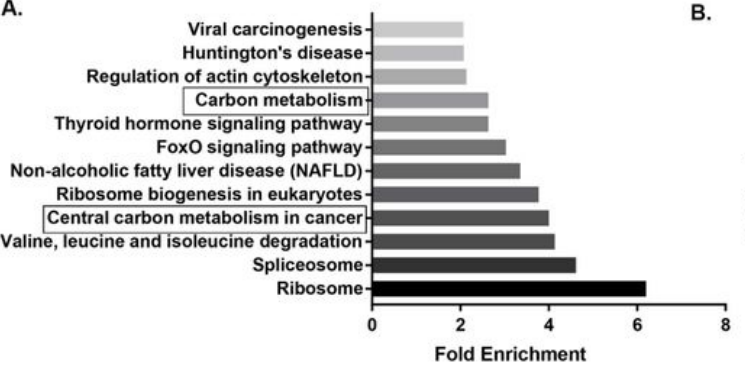

c.
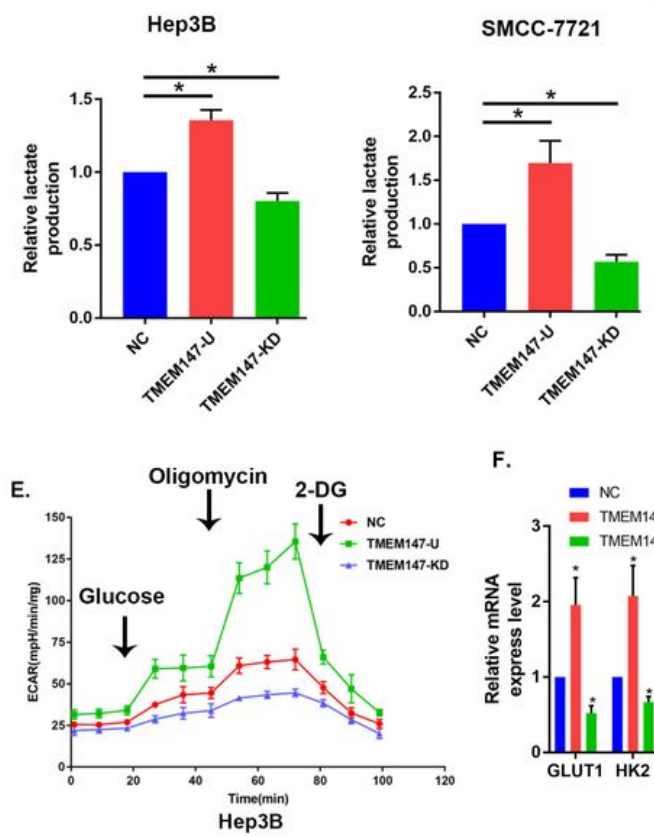

D.

F.
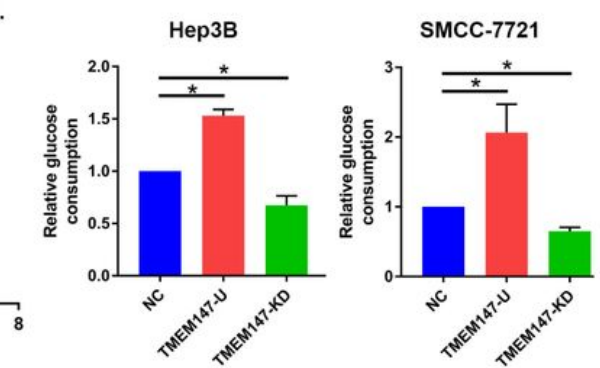
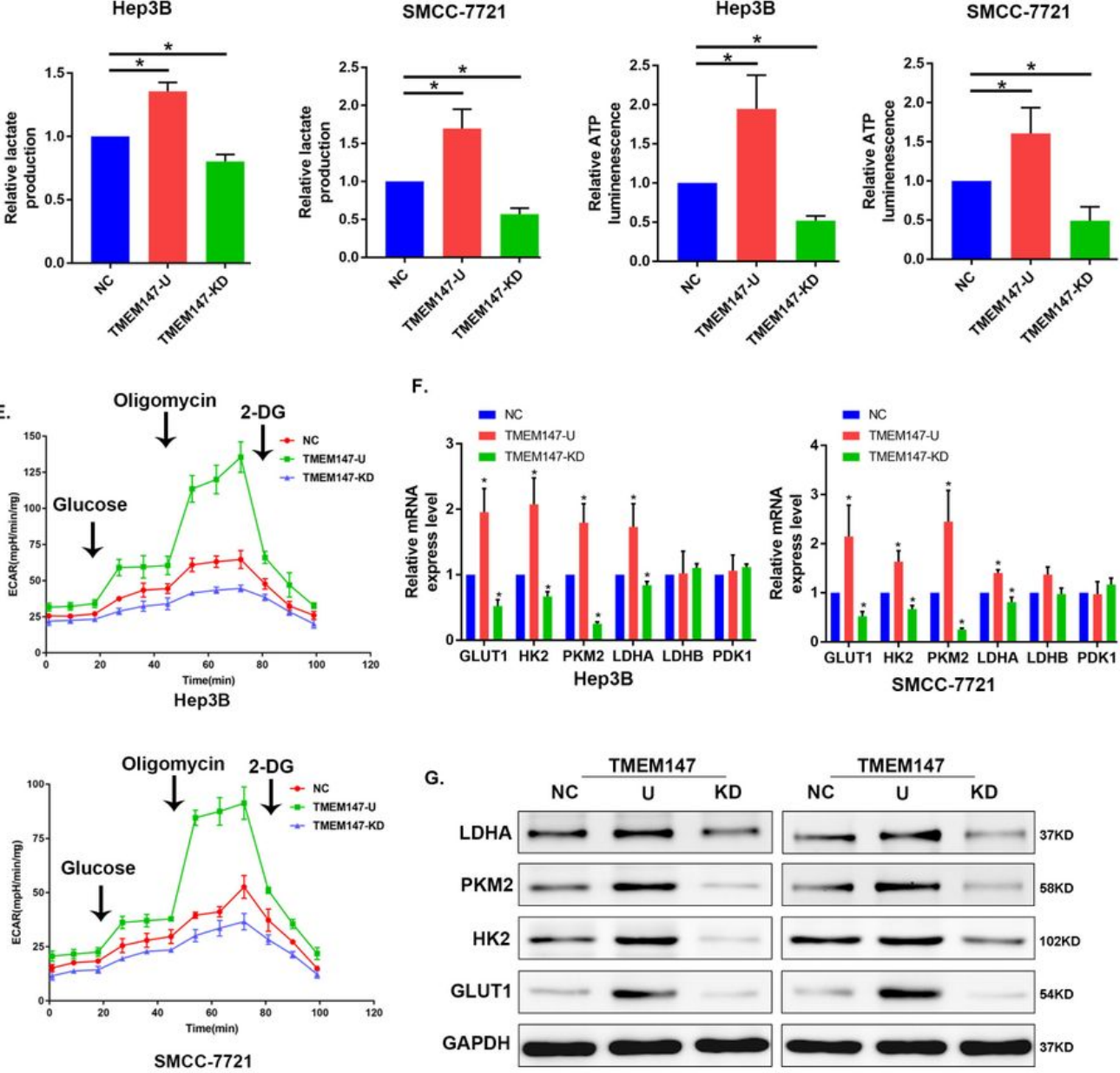

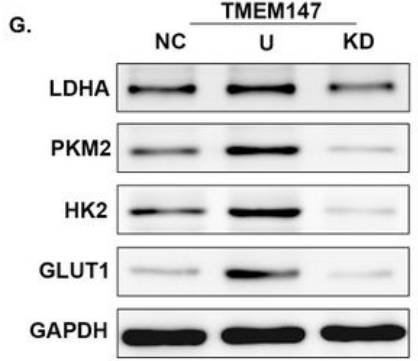

Hep3B

SMCC-7721

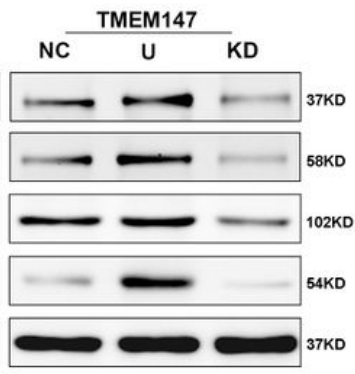

SMCC-7721

Figure 3

Effects of TMEM147 on glycolysis in HCC cells a. GO enrichment analysis of TMEM147 downstream pathway based on LIHC in TCGA database. b-d. Glucose consumption (b), lactate production (c), ATP production (d), and ECAR (e) were measured in TMEM147-overexpressing and -knockdown HCC cells. f-g. Expression levels of several metabolic enzymes (GLUT1, PKM2, HK2, LDHA) in TMEM147-overexpressing and -knockdown HCC cells determined by RT-qPCR and western blot analysis. ${ }^{*} p<0.05$ was regarded as statistically significant. 
A.

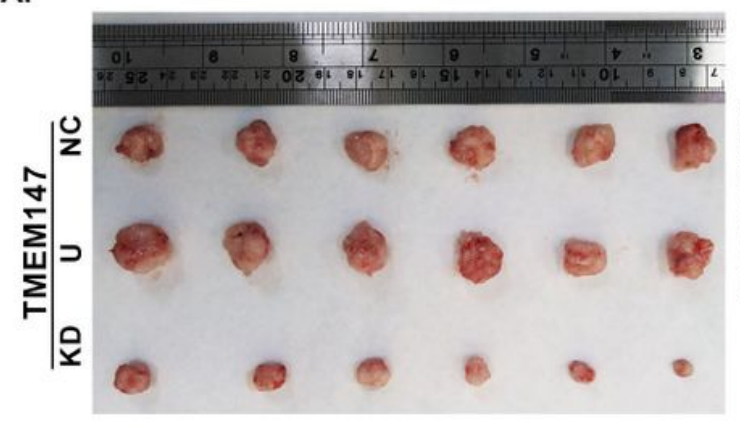

B.

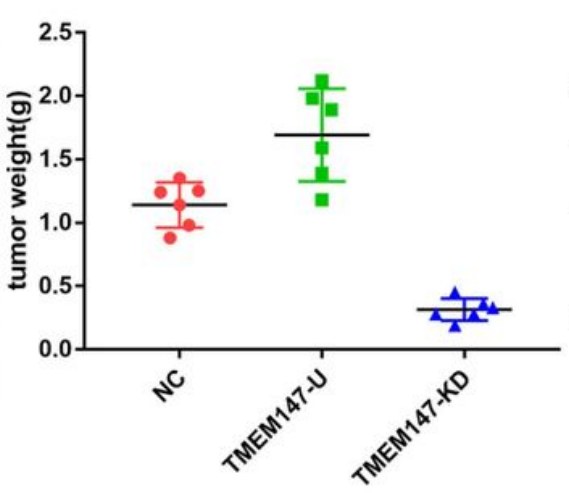

c.

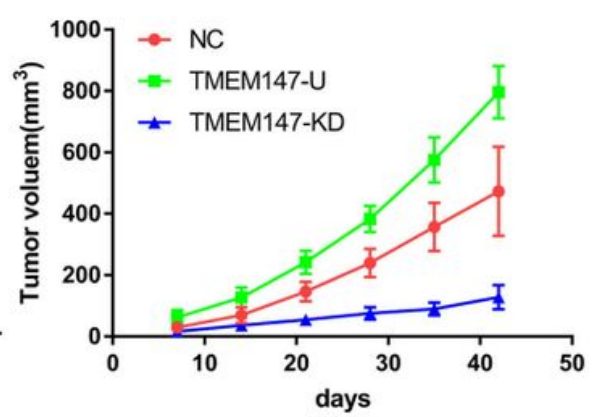

D.

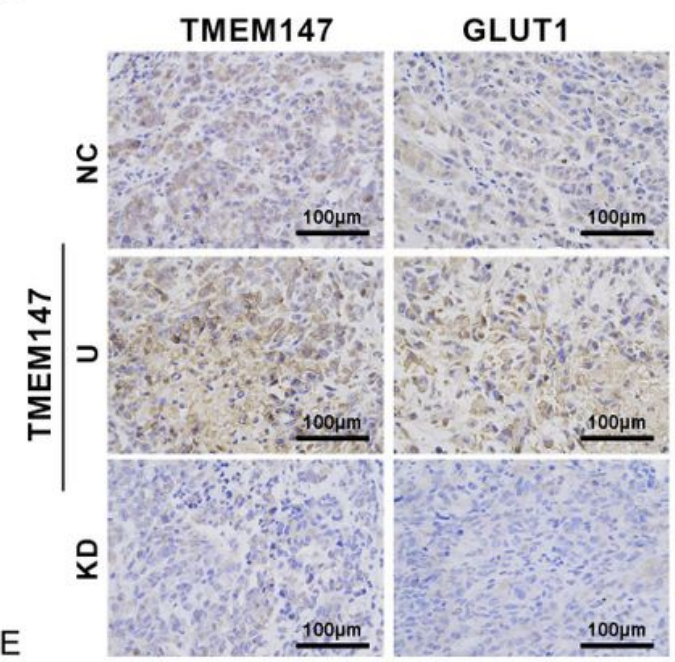

NC
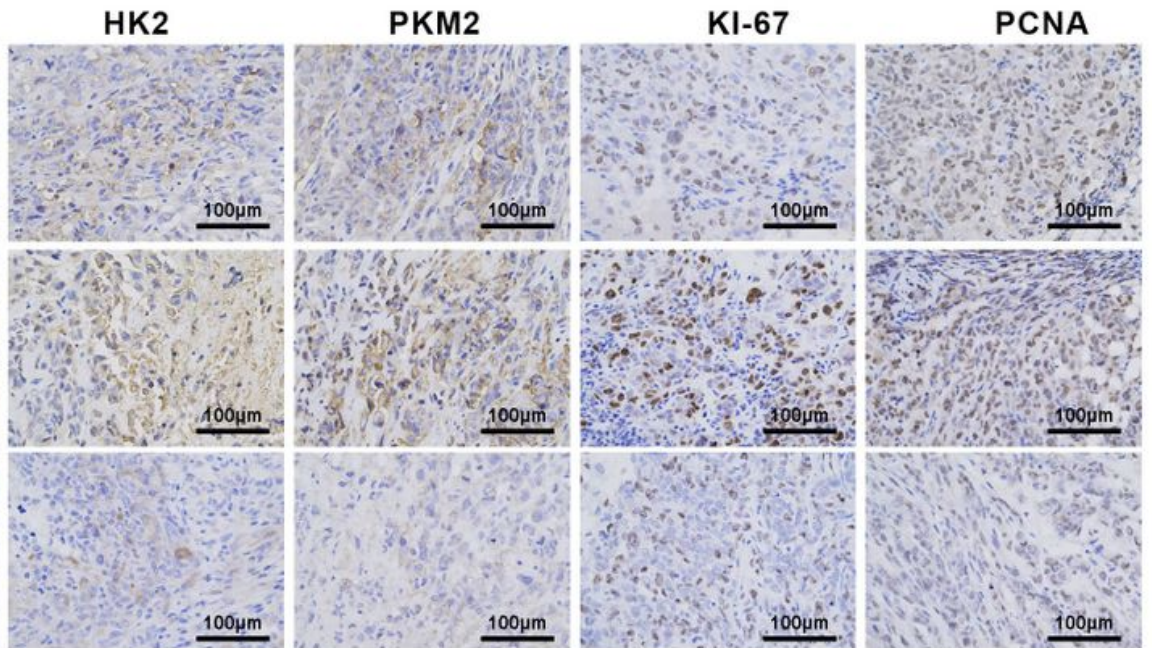

TMEM147-U

TMEM147-KD
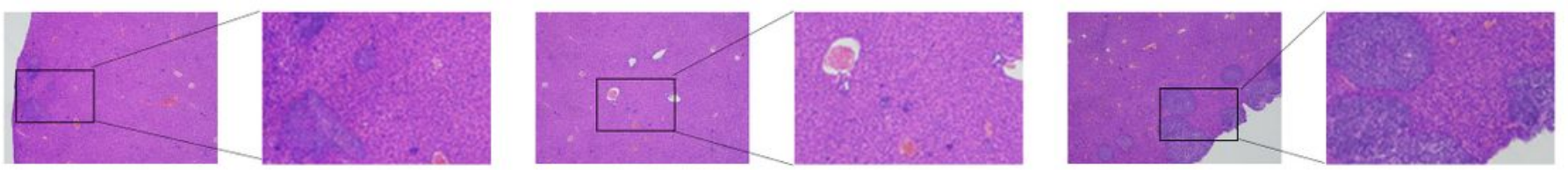

Figure 4

Effects of TMEM147 on HCC growth in vivo A. Representative image of nude mouse tumors $(n=6)$. B, C. Implanted tumor weight (B) and volume (C) of TMEM147-overexpressing and -knockdown tumors. D. Typical IHC staining images showing expression of $\mathrm{Ki}-67$, proliferating cell nuclear antigen, and glycolysis enzymes in transplanted tumors under different experimental conditions. E. Representative H\&E staining and quantification of metastatic hepatic nodules of TMEM147 overexpressed and knockdown group. * $p<0.05$ was regarded as statistically significant. 
- - D

TMEM147

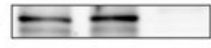

Нерзв

SMMC-7721
DAPI
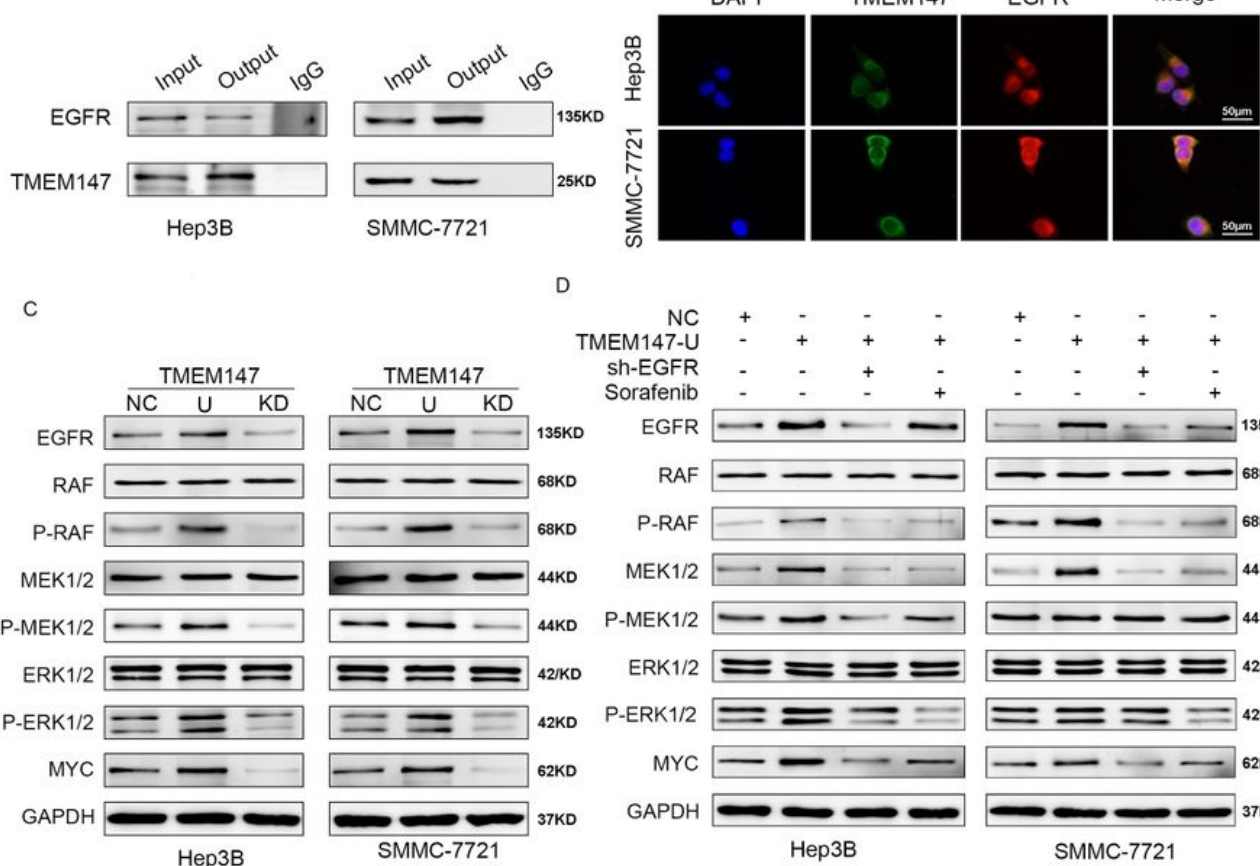

SMMC-7721

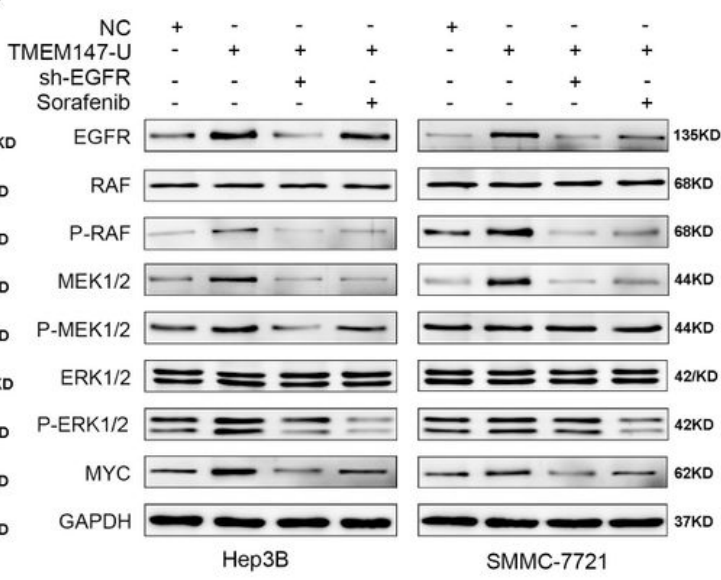

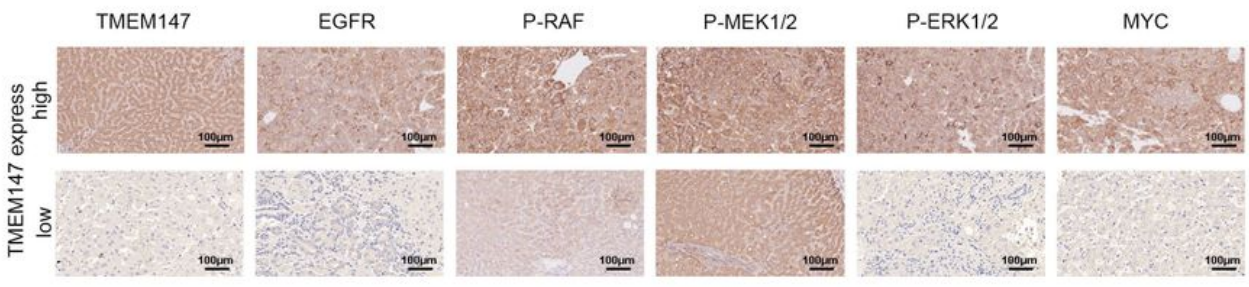
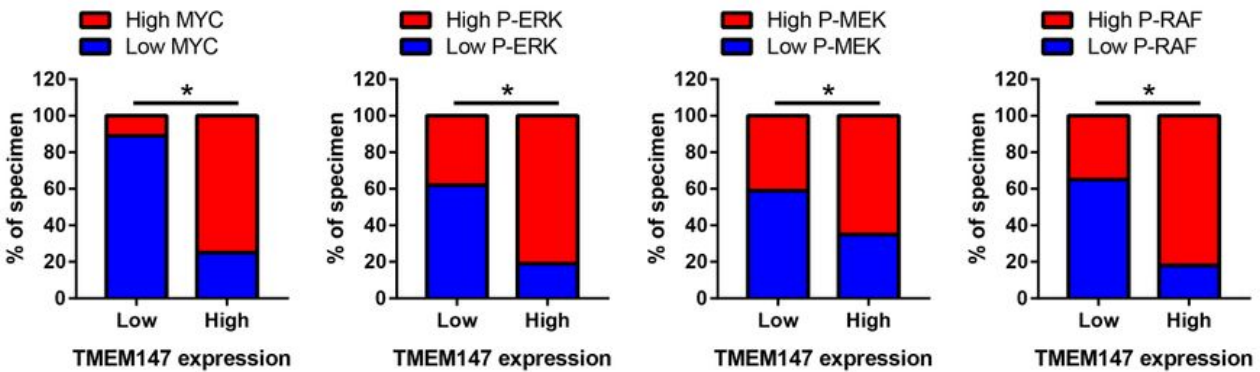

\section{Figure 5}

Interaction between TMEM147 and EGFR and effect on MAPK signaling A. Immunoprecipitation assay analysis the interaction of TMEM147 and EGFR. B. Immunofluorescence analysis of the colocalization of TMEM147 and EGFR in the cell subcellular subcellular organelle. C. Expression levels of EGFR expression and MAPK activity in TMEM147-

overexpressing and -knockdown HCC cells determined by Western blot analysis. D. EGFR shRNA or sorafenib could reverse the effect of TMEM147 overexpression on EGFR expression and MAPK activity in HCC cells. E. IHC analysis the correlation of TMEM147 with the EGFR, p-RAF, p-MEK, p-ERK, MYC expression. ${ }^{*} \mathrm{p}<0.05$ was regarded as statistically significant. 

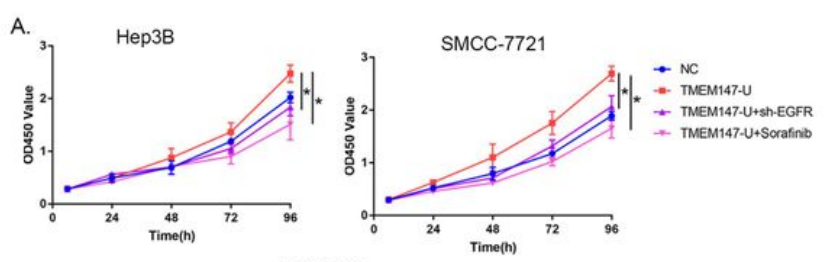

C.
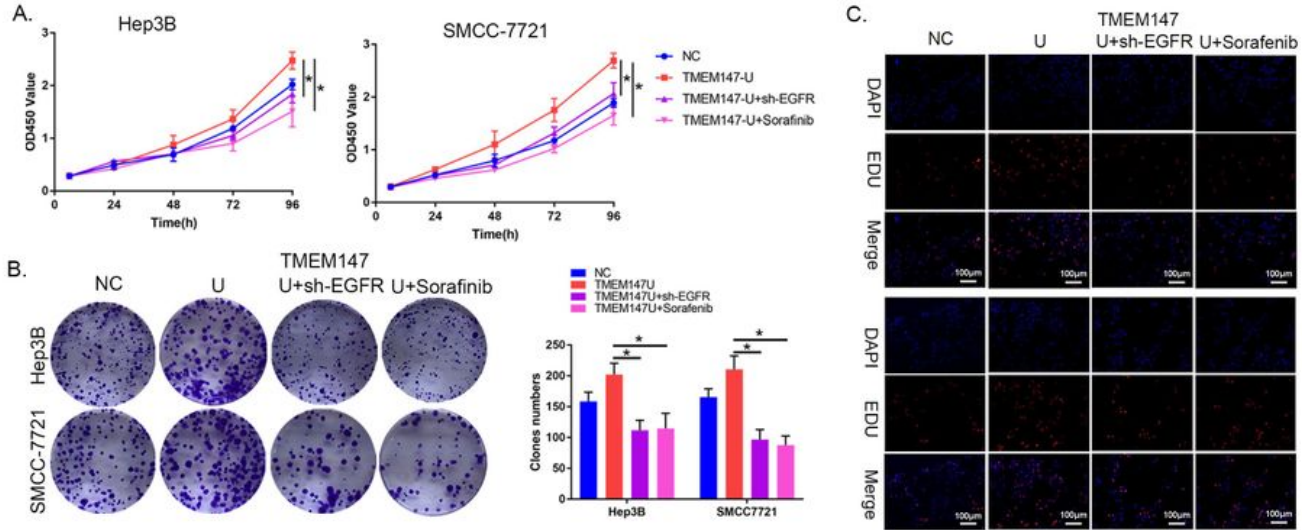

D.

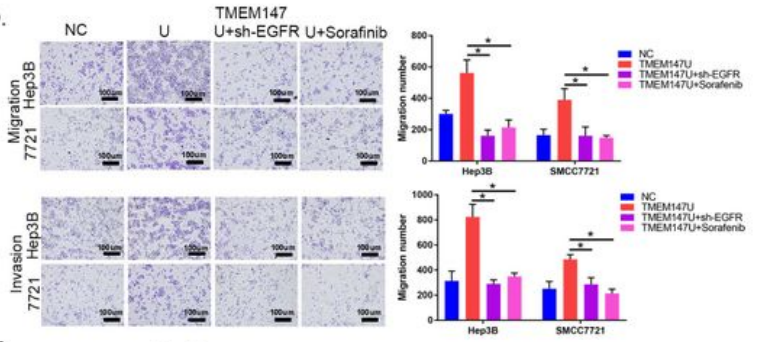

E.

Нерзв

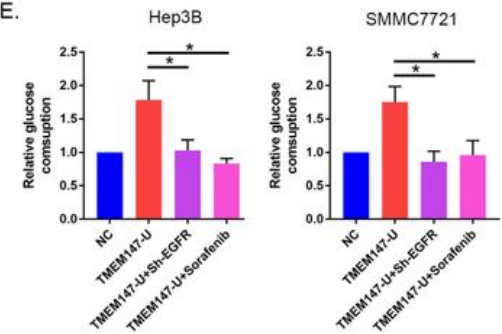

F.

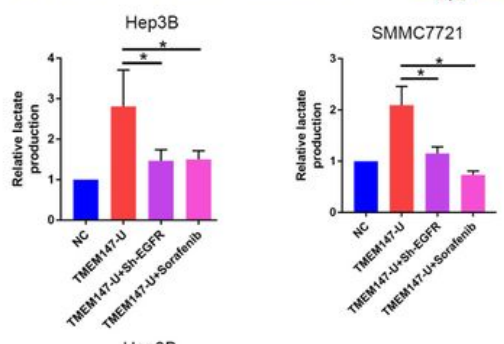

G.
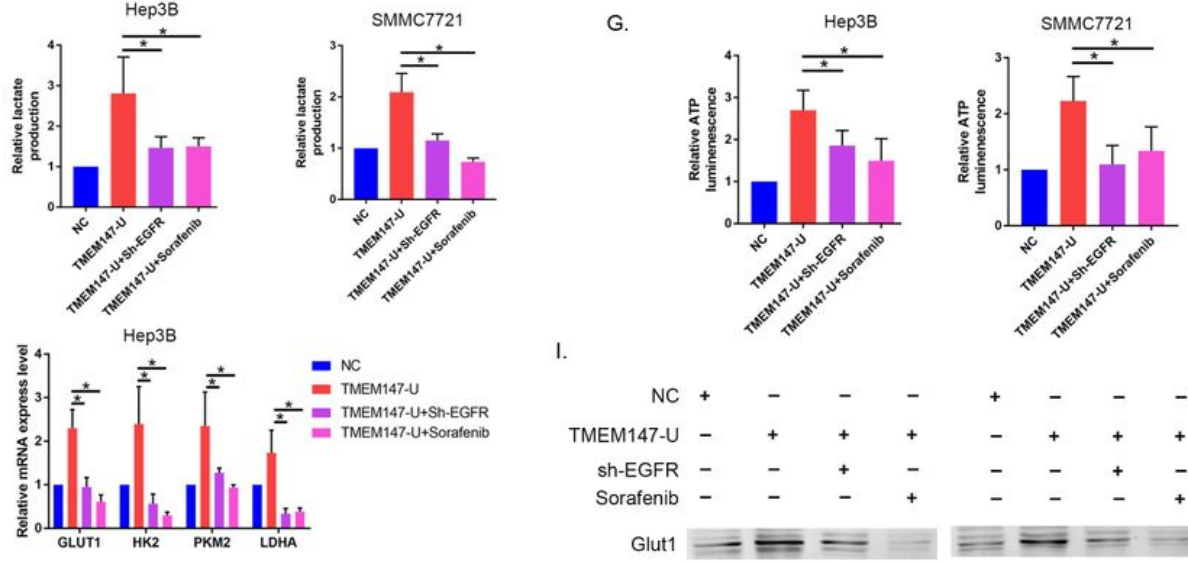

I.
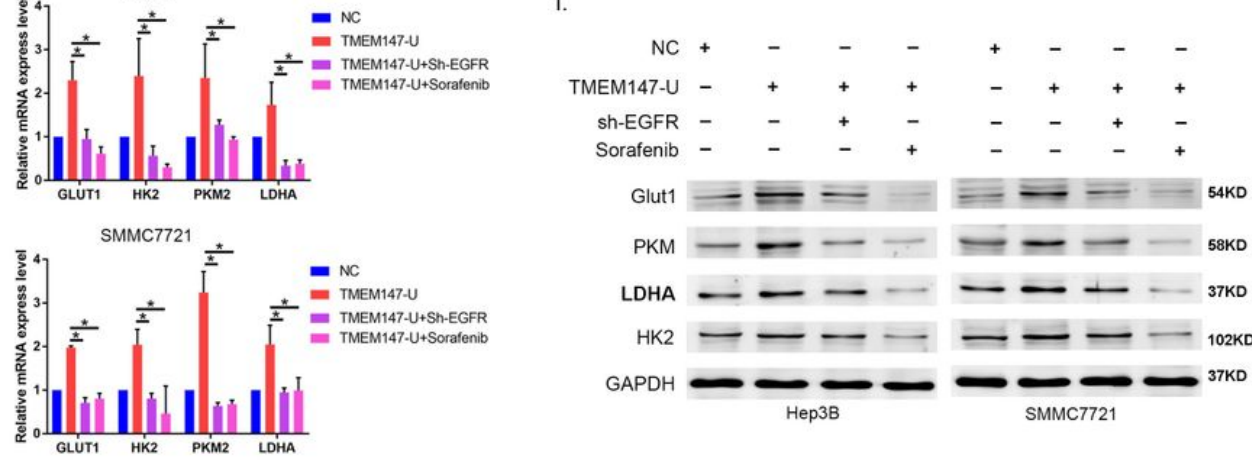

Figure 6

TMEM147 drives HCC tumorigenesis via EGFR A-C. Effects of EGFR shRNA and sorafenib on proliferation of TMEM147overexpressing cells determined by CCK8, EDU, and colony-formation assays. D, E. EGFR shRNA or sorafenib could partly inhibit the migration and invasion of TMEM147-overexpressing HCC cells, shown by transwell migration and invasion assays. F-H. EGFR shRNA or sorafenib could reverse the effect of TMEM147 overexpression on glycolysis in HCC cells, demonstrated by glucose consumption (F), lactate production (G), and ATP production $(\mathrm{H})$ assays. I, J. Effects of EGFR shRNA or sorafenib on TMEM147-induced expression of key glycolysis enzymes. ${ }^{*} p<0.05$ was regarded as statistically significant. 


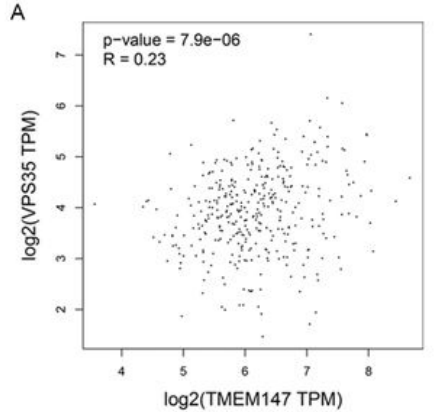

B
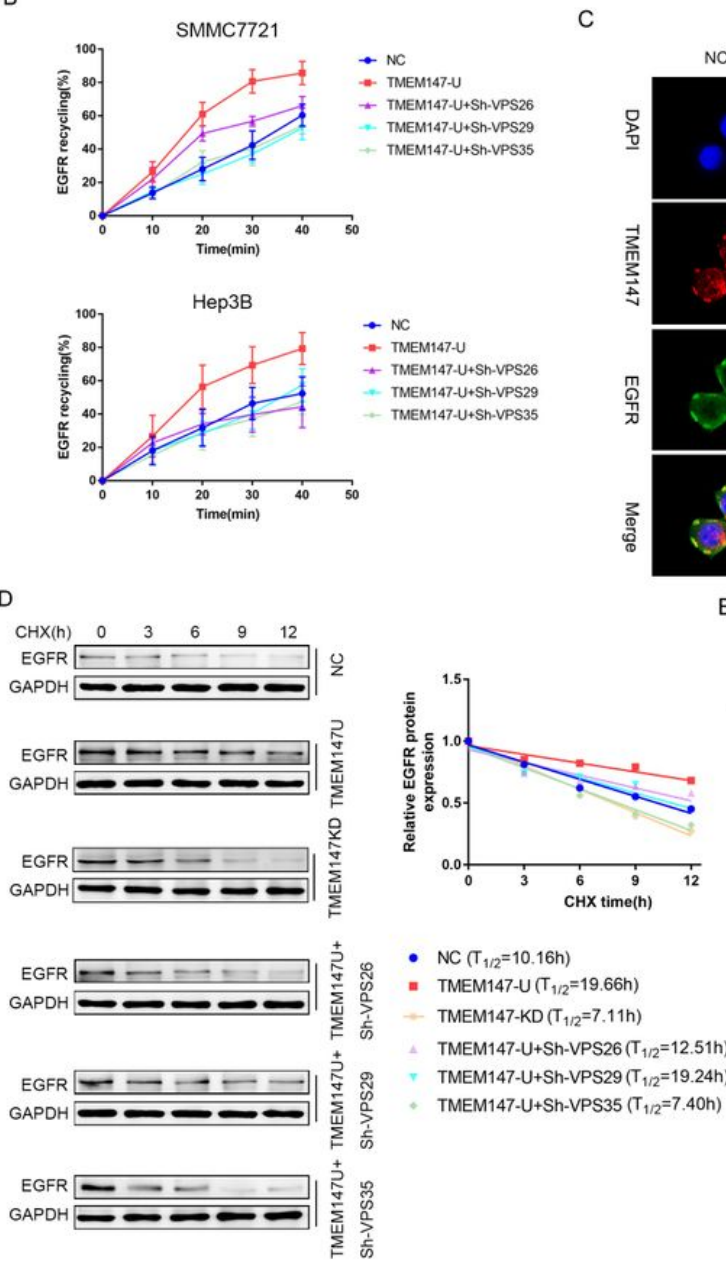

C
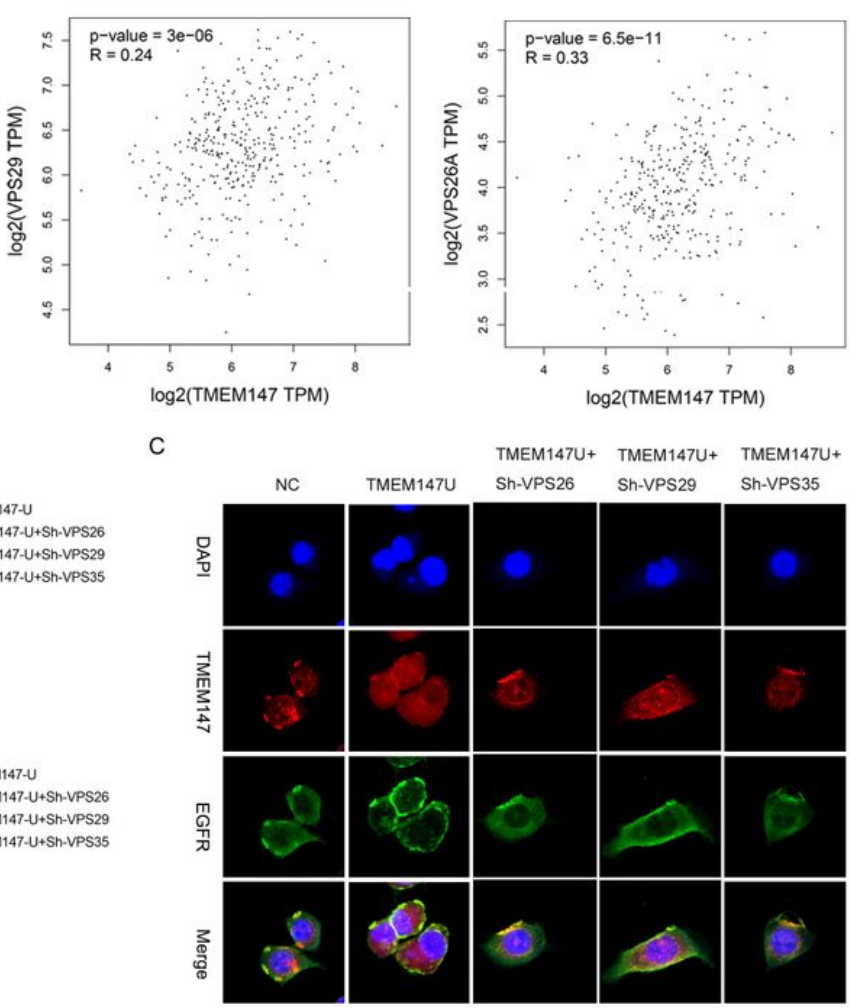

E

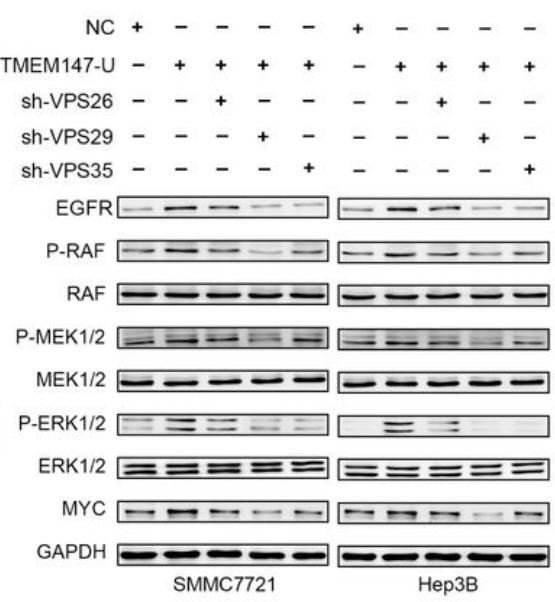

\section{Figure 7}

Role of retromer as a platform for TMEM147-induced EGFR/MAPK pathway A. Bioinformatics analysis of correlations between TMEM147 and retromer structural (VPS26, VPS29, VPS35) in LIHC in TCGA database. B. Effects of VPS26, VPS29, and VPS35 downregulation on EGFR recycling in TMEM147-overexpressing cells determined by ELISA recycling assay. C. Immunofluorescence assay analysis of the EGFR recycle. D. Effects of VPS26, VPS29, and VPS35 downregulation on EGFR degradation in TMEM147-overexpressing cells determined by protein stability assay. E. Effects of VPS26, VPS29, and VPS35 downregulation on expression and activity of EGFR downstream MAPK pathway in TMEM147overexpressing cells determined by western-blot assay. ${ }^{*} p<0.05$ was regarded as statistically significant. 
A.
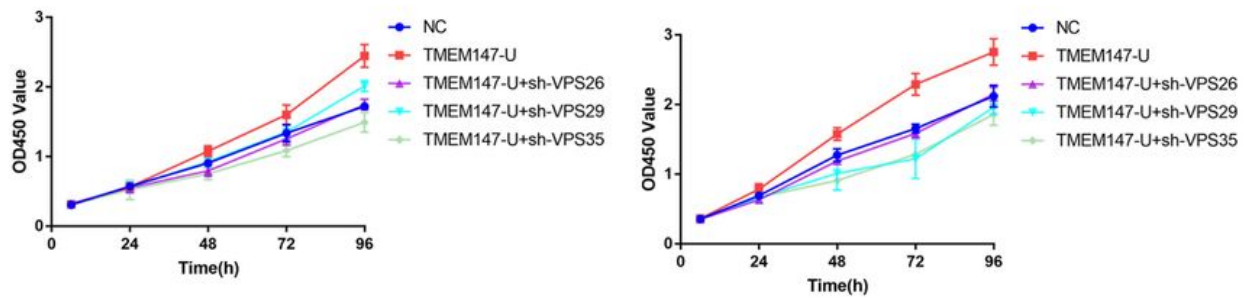

B.
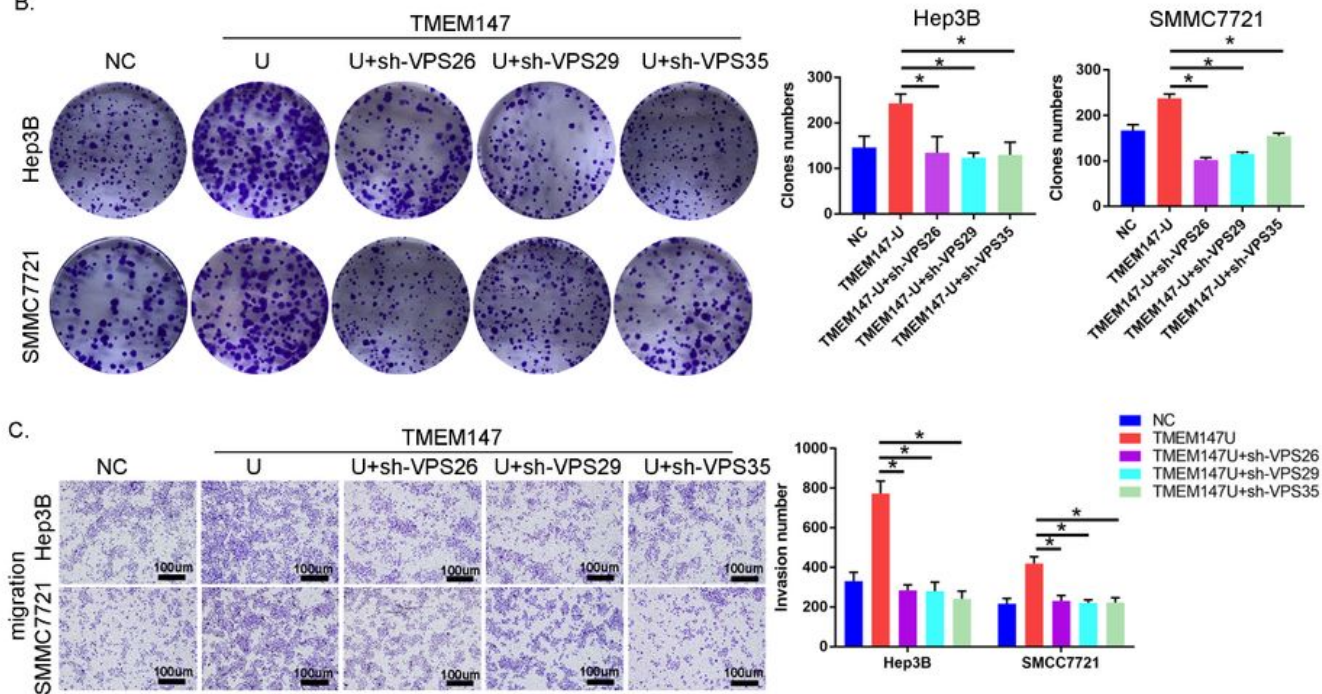

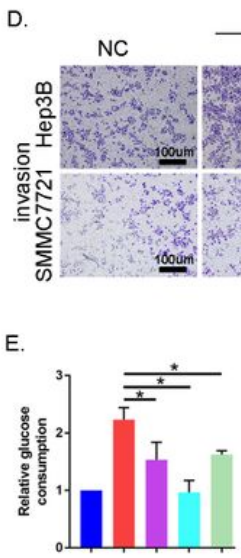

Hep3B

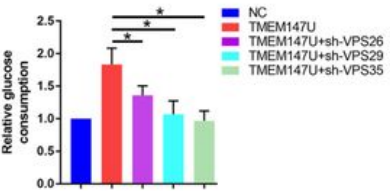

SMMC7721

TMEM147

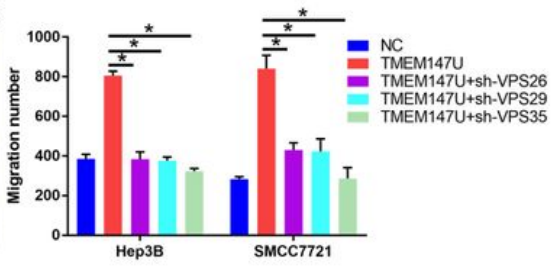

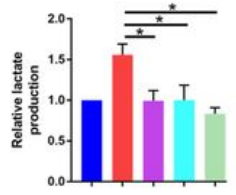

Нер3в

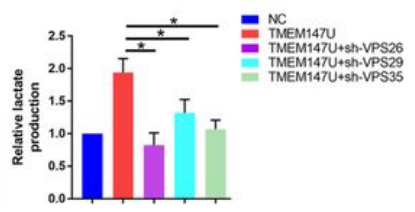

SMMC7721

\section{Figure 8}

Effects of TMEM147 on HCC proliferation, invasion, and glycolysis via retromer components A, B. HCC cell proliferation in the indicated groups, according to CCK8 and colony-formation assays. C, D. Migration and invasion of HCC cells in the indicated groups, according to transwell assays. E-F. Glycolysis effects in the indicated groups, determined by cellular glucose uptake and lactate production assays. The indicated groups were negative control (NC), TMEM147-upregulated (TMEM147-U), TMEM147-upregulated combined with knockdown of VPS26 (TMEM147U+sh-VPS26), VPS29 (TMEM147U+sh-VPS29), and VPS35 (TMEM147U+sh-VPS35). * $<0.05$ was regarded as statistically significant.

\section{Supplementary Files}

This is a list of supplementary files associated with this preprint. Click to download.

- FigurerS1.tif 
- FigureS2.tif

- FigureS3.tif

Page 22/22 